\title{
Coarse and synthetic Weil-Petersson geometry: quasi-flats, geodesics and relative hyperbolicity
}

\author{
JEFFREY BROCK \\ HOWARD MASUR
}

\begin{abstract}
We analyze the coarse geometry of the Weil-Petersson metric on Teichmüller space, focusing on applications to its synthetic geometry (in particular the behavior of geodesics). We settle the question of the strong relative hyperbolicity of the WeilPetersson metric via consideration of its coarse quasi-isometric model, the pants graph. We show that in dimension 3 the pants graph is strongly relatively hyperbolic with respect to naturally defined product regions and show any quasi-flat lies a bounded distance from a single product. For all higher dimensions there is no nontrivial collection of subsets with respect to which it strongly relatively hyperbolic; this extends a theorem of Behrstock, Druţu and Mosher [2] in dimension 6 and higher into the intermediate range (it is hyperbolic if and only if the dimension is 1 or 2 by Brock and Farb [7]). Stability and relative stability of quasi-geodesics in dimensions up through 3 provide for a strong understanding of the behavior of geodesics and a complete description of the CAT(0)-boundary of the Weil-Petersson metric via curve-hierarchies and their associated boundary laminations.
\end{abstract}

30F60; 20F67

\section{Introduction}

The study of the large scale geometry of Teichmüller space and has given rise to new perspectives on Teichmüller geometry and dynamics in recent years, with results of Masur-Minsky [20], Brock [5], Brock-Farb [7], Behrstock-Minsky [3], Rafi [22], Wolpert [25] and others giving insight into coarse phenomena that arise in consideration of various metrics. Notable among these is the Weil-Petersson metric on Teich $(S)$, which carries a convenient coarse description in terms of combinatorics of pants decompositions of a surface [5].

One feature that is common to virtually all these investigations is the emergence of obstructions to hyperbolicity (in the sense of Gromov) in higher dimensional cases the Weil-Petersson metric is Gromov hyperbolic if and only if the Teichmüller space has dimension at most 2 [7]. When a space fails to be Gromov hyperbolic, the lack of 
stability properties familiar in negative curvature impedes an immediate understanding of the behavior of geodesics and quasi-geodesics.

Nevertheless, the notion of strong relative hyperbolicity allows for similar control of quasi-geodesics up to their behavior in certain well defined regions that are coarsely isolated from one another. In this paper we flesh out precisely in which cases the Weil-Petersson metric exhibits such strong relative hyperbolicity.

It is important to note that understanding the coarse, large scale structure of a metric space can lead to a precise understanding of fine structure in the setting of CAT(0) and NPC (nonpositively curved) geometry. As an example, our methods provide for a complete description of the CAT( 0$)$ boundary of the Weil-Petersson metric up through Teichmüller spaces of dimension 3 , originating out of a purely coarse combinatorial model.

Let $S=S_{g, n}$ be a compact surface of genus $g$ with $n$ boundary components. We define the complexity $\zeta(S)$ of $S$ to be the integer $3 g-3+n$, namely, the complex dimension of the corresponding Teichmüller space Teich $(S)$, or the Teichmüller dimension of $S$. The initial focus of the paper will be on the cases $(g, n) \in\{(2,0),(1,3),(0,6)\}$. We say a curve $\gamma$ is domain separating if $S \backslash \gamma$ has two components neither of which is a three-holed sphere.

A domain separating curve $\gamma$ on $S$ determines a set $X_{\gamma} \subset P(S)$ consisting of pants decompositions that contain $\gamma$. When $\zeta(S)=3$ and $\gamma$ is domain separating, the set $X_{\gamma}$ naturally decomposes as a product of Farey graphs, each naturally the pants graph on the complementary one-holed torus or four holed sphere in $S \backslash \gamma$. We show the following.

Theorem 1 Let $S=S_{g, n}$ where $(g, n) \in\{(2,0),(1,3),(0,6)\}$. Then the pants graph $P(S)$ is strongly relatively hyperbolic relative to the sets $X_{\gamma}$ where $\gamma$ ranges over all domain separating curves in $S$.

For the purposes of the proof, we will refer to the formulation of strong relative hyperbolicity given by Druţu and Sapir [11] and refined by Druţu [10].

Roughly speaking, relative hyperbolicity guarantees that by "coning-off" each $X_{\gamma}$ to a single point $p_{\gamma}$ by edges of length one, the resulting metric is Gromov hyperbolic. The theorem asserts further that this relative hyperbolicity is strong in the sense that the subsets $X_{\gamma}$ satisfy the bounded region (or coset) penetration property (cf [13; 7]).

In particular, this condition implies that when two quasi-geodesics in $P(S)$ begin and end at the same position, they enter and exit uniform neighborhoods of each $X_{\gamma}$ within 
a bounded distance of one another. We give a more formal definition in Section 3 and prove the requisite bounded coset penetration property in Proposition 1.

Our considerations have been motivated by the notion of a hierarchy path, a particular kind of quasi-geodesic arising out of the hyperbolicity of the curve complex. In MasurMinsky [20], this transitive family of quasi-geodesics in $P(S)$ is described, built up from geodesics in the curve complexes of nonannular essential subsurfaces of $S$. Such a quasi-geodesic is called a resolution of a hierarchy $H\left(P_{1}, P_{2}\right)$ connecting $P_{1}$ and $P_{2}$ in $P(S)$.

In the above cases, given a resolution $\rho:[0, n] \rightarrow P(S)$ of a hierarchy $H\left(P_{1}, P_{2}\right)$ we denote by $X(\rho)$ the union of the image of $\rho$ and the Farey-graph products $X_{\gamma}=$ $P(W) \times P\left(W^{c}\right)$ where $\gamma \in \mathcal{C}(S)$ is the common boundary of $W$ and $W^{c}$ for which either $W$ or $W^{c}$ is a "component domain" of $H\left(P_{1}, P_{2}\right)$ [20].

Then Theorem 1 will follow from the following quasi-convexity result.

Theorem 2 For any resolution $\rho$ of the hierarchy $H\left(P_{1}, P_{2}\right)$, the union $X(\rho)$ is quasi-convex in $P(S)$.

Quasi-convexity of this set guarantees that quasi-geodesics in $P(S)$, while not stable in the whole of $P(S)$, do satisfy a relative stability with respect to the product regions $X_{\gamma}$.

Rank and quasi-flats A quasi-flat $F$ in a metric space $X$ is a quasi-isometric embedding $^{1}$

$$
F: \mathbb{R}^{n} \rightarrow X
$$

where $n \geq 2$. The integer $n$ is called the rank of the quasi-flat. The investigations due to Kleiner and Leeb [17] and Eskin and Farb [12] of quasi-isometric rigidity in the setting of higher rank symmetric spaces each used the classification of quasi-flats as a central tool.

Let $\zeta(S)=3$, and let $\gamma$ be a separating curve for which each complementary $X_{1}$ and $X_{2}$ satisfies $\zeta\left(X_{i}\right)=1$. Then the Farey-graph product $P\left(X_{1}\right) \times P\left(X_{2}\right)$ sits naturally in $P(S)$ as the subset $X_{\gamma} \subset P(S)$ consisting of pants decompositions containing the curve $\gamma$. Theorem 2 then allows one to give a classification of maximal quasi-flats for $P(S)$ in the cases when $\zeta(S)=3$.

\footnotetext{
${ }^{1}$ The map $F$ is a quasi-isometric embedding if $F$ distorts distances by a bounded additive and multiplicative amount.
} 
Theorem 3 (Quasi-flats theorem) Let $\zeta(S)=3$. Then each quasi-flat $F$ in $P(S)$ has rank 2 and lies a bounded distance from a product $P\left(X_{1}\right) \times P\left(X_{2}\right) \subset P(S)$ of Farey graphs corresponding to the complementary subsurfaces of a domain-separating curve $\gamma$.

The geometric rank of a metric space $X$ is the maximal positive integer $n$ for which $X$ admits a quasi-isometric embedding $F: \mathbb{R}^{n} \rightarrow X$. As a consequence we obtain the following corollary, verifying a conjecture of the first author and Farb [7] in the case $\zeta(S)=3$. We remark that the following statement has been obtained independently (and for all surfaces $S$ ) by work of Behrstock and Minsky [3].

Corollary 4 (Geometric rank) When $\zeta(S)=3$ the geometric rank of the pants graph $P(S)$ and hence the Weil-Petersson metric on $\operatorname{Teich}(S)$ is 2 .

The boundary of the Weil-Petersson metric Our main theorem has applications for understanding the CAT(0) geometry of the Weil-Petersson metric.

The Weil-Petersson metric on the Teichmüller space $\operatorname{Teich}(S)$ has negative curvature, but it is not complete. Its completion $\overline{\operatorname{Teich}(S)}$ has the structure of a CAT( 0$)$ space, namely, a geodesic metric space $X$ in which pairs of points on edges of a geodesic triangle have distance at most that of the distance between corresponding points on a triangle in Euclidean space.

It is shown by the first author [6] that the unit tangent spheres have no natural identification. However, the notion of an asymptote class of infinite geodesic rays is natural and basepoint independent. When $X$ is Gromov hyperbolic, this CAT(0) boundary agrees with the usual Gromov boundary.

As a consequence of Theorem 2 and the main result of Brock-Farb [7], we give a description of the CAT( 0$)$ boundary of the Weil-Petersson metric when $3 g-3+n \leq 3$.

We say $\lambda$ is a boundary lamination if each component $\lambda^{\prime}$ of $\lambda$ is a Gromov boundary point of $\mathcal{C}\left(S\left(\lambda^{\prime}\right)\right)$ where $S\left(\lambda^{\prime}\right)$ represents the minimal subsurface of $S$ containing $\lambda^{\prime}$. There is a natural topology on boundary laminations, which we formulate in Section 4 , but we warn the reader in advance that it is not continuous with respect to usual topologies on laminations arising out of consideration of transverse measures.

Theorem 5 Let $S_{g, n}$ satisfy $3 g-3+n \leq 3$. Then the CAT(0) boundary of the WeilPetersson metric on Teich $(S)$ is homeomorphic to the space of boundary laminations. 
We remark that both the notion of a boundary lamination defined here as well as the appropriate topology on the space of such depend on the strong characterization of quasi-geodesics of Theorem 2. Thus, with our methods, such a discussion is specific to dimension at most 3. In a separate paper with Minsky, we develop a general notion of a lamination associated to a Weil-Petersson geodesic ray that arises from convexity of length functions along geodesics [8].

In the present discussion, the principal idea from Theorem 2 that infinite rays in the Weil-Petersson metric have associated "infinite hierarchies without annuli" in the sense of Masur and Minsky [20] gives rise to a natural way to associate infinite geodesics in the curve complex of $S$ or its subsurfaces. Indeed, the set of curves on $S$ used to construct "hierarchies" has infinite diameter in the curve complex of some subsurface of $Y$ of $S$. When there is a unique such $Y$, the hierarchy is essentially determined by the asymptotic data of a point in the Gromov boundary $\partial \mathcal{C}(Y)$. In the case at hand, the only possibility if there is more than one such $Y$ is that there are two such subsurfaces $Y$ and $Y^{c}$, and the hierarchy in question has infinite diameter in each. In this case the related rate of divergence of the geodesic in each factor $\mathcal{C}(Y)$ and $\mathcal{C}\left(Y^{c}\right)$ determine an additional piece of data, the "slope" of the divergence of the ray. To encode this slope we associate real weights to the two laminations and projectivize. We will describe this in more detail in Section 4.

Thickness and relative hyperbolicity In the paper [7], the first author and Farb showed that for each $S$ with $\zeta(S) \geq 3$ the pants graph $P(S)$, and thence the WeilPetersson metric on Teich $(S)$, is not Gromov hyperbolic. As the central obstruction to hyperbolicity is the existence of quasi-isometrically embedded product regions, one can ask whether a line of reasoning similar to the above approach to the case $\zeta(S)=3$ might persist in higher complexity.

Behrstock, Drutzu and Mosher [2] take up this theme in generality; the notion of a thick metric space is introduced, and it is shown that for $\zeta(S) \geq 6$ the pants graph $P(S)$ is thick. This condition is equivalent $[2 ; 11]$ to the failure of strong relatively hyperbolicity in the sense of Druţu and Sapir [11].

The argument given in [2] for the thickness of the Weil-Petersson metric on Teichmüller space runs aground in the cases of mid-range complexity, namely $\zeta(S)=4$ and 5 . Pushing their approach a bit further we show that our strong relative hyperbolicity theorem for $\zeta(S)=3$ is sharp in the following sense.

Theorem 6 Let $S$ be a surface with $\zeta(S) \geq 4$. Then pants graph $P(S)$ is not strongly relatively hyperbolic with respect to any co-infinite collection of subsets. 
Here, a subset $Y \subset X$ of a metric space $Y$ is co-infinite if there are points in $X$ at arbitrarily large distance from $Y$. The theorem is a consequence of the fact that in these cases the pants graph is thick in the sense of [2], shown in Theorem 18.

The theorem extends the relevant result of [2] which treats the case $\zeta(S) \geq 6$, to the intermediate range $\zeta(S) \in\{4,5\}$ and establishes the failure of strong relative hyperbolicity with respect to any collection of co-infinite subsets in general for $\zeta(S) \geq 4$.

Theorem 6 shows that the precise control of geodesics using these coarse methods stops in Teichmüller dimension $3(\zeta(S)=3)$. Interestingly, the central feature of these non-relatively hyperbolic cases is the ability to "chain flats" in a gross sense: roughly speaking one can join any pair of points without ever leaving a union of quasiisometrically embedded copies of $\mathbb{R}^{2}$. While this can be done as early as dimension 2 for the mapping class group, this kind of connectivity only begins in dimension 4 .

Plan of the paper We begin with preliminaries, condensing the notions required from the coarse geometry of the curve complex into the manageable formulation of a hierarchy path, namely, a particular type of quasi-geodesic in $P(S)$ arising inductively from the hyperbolicity of the curve complex. In Section 3, we show that when $\zeta(S)=3$ we have relative stability of quasi-geodesics in $P(S)$. In Section 4, we deduce applications of this stability result to the finer structure of the CAT(0) boundary of the Weil-Petersson metric for $S$ with $\zeta(S) \leq 3$. Finally, in Section 5 we exhibit the "thickness" of the pants graph $P(S)$ and thence the Weil-Petersson metric for $S$ with $\zeta(S)=4$ and 5 and discuss how it follows naturally that the Weil-Petersson metric cannot be strongly relatively hyperbolic with respect to any collection of coinfinite subsets for $\zeta(S) \geq 4$. This final result shows that hyperbolicity and strong relative hyperbolicity, and the concomitant synthetic control, are sharp to Teichmüller dimension at most 3 .

Acknowledgments The authors gratefully acknowledge the support of the many institutions that have played a role in supporting this research including the University of Chicago and the University of Texas at Austin. The second author thanks Brown University for its hospitality while this work was being completed. The authors also thank Jason Behrstock, Cornelia Druţu, Benson Farb, Lee Mosher and Mark Sapir for many useful and illuminating conversations and comments. The authors would also like to thank the referee for several clarifying comments. This work has some thematic overlap with our forthcoming joint project with Yair Minsky [8], whom we also thank for his continued support and collaboration. 


\section{Preliminaries}

In this section we set out some of the preliminary notions we will need.

Complexes of curves and pants Let $S$ be a compact surface of negative Euler characteristic. The curve complex of $S$, denoted $\mathcal{C}(S)$, is the simplicial complex whose vertices correspond to isotopy classes of essential simple closed curves on $S$ and whose $k$-simplices span collections of $k+1$ vertices whose corresponding simple closed curves can be realized pairwise-disjointly by simple closed curves on the surface. We will be primarily interested in the 1 -skeleton of $\mathcal{C}(S)$, often called the curve graph of $S$, and the associated distance function $d_{S}(.,$.$) on the 0$-skeleton induced by the metric obtained by assigning each edge length 1 .

A related notion, the pants graph $P(S)$ associated to $S$ is a graph whose vertices correspond to pants decompositions, namely, maximal families of distinct, essential, nonperipheral isotopy classes of simple closed curves on $S$ so that the classes in the family have pairwise disjoint representatives.

In this case, edges connect vertices whose corresponding pants decompositions differ by an elementary move: pants decompositions $P$ and $P^{\prime}$ differ by an elementary move if $P^{\prime}$ can be obtained from $P$ by replacing one isotopy class $\alpha$ in $P$ by another $\beta$ so that representatives of $\beta$ intersect representatives of $\alpha$ minimally among all possible choices for $\beta$. These moves have two types (see Figure 1). In the first type $P \backslash \alpha$ contains a torus with a hole and $\beta$ intersects $\alpha$ once. In the second $P \backslash \alpha$ contains a 4 holed sphere and $\beta$ intersects $\alpha$ twice.

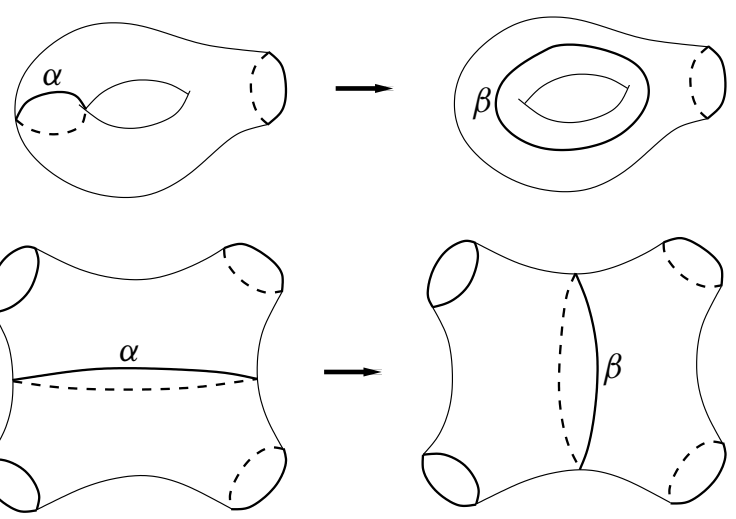

Figure 1: Elementary moves on pants decompositions 
It is a theorem of Hatcher and Thurston that the graph $P(S)$ is connected [14], and thus there is a notion of distance between vertices obtained by metrizing $P(S)$ so that each edge has length 1 . We denote by $d(\cdot, \cdot)$ this distance on $P(S)$.

Given a subsurface $Y \subseteq S$ that is not an annulus, work of Masur and Minsky [20] define a coarse projection $\pi_{Y}$ from $\mathcal{C}(S)$ to uniformly bounded diameter subsets of $\mathcal{C}(Y)$, as follows.

If $\gamma \in \mathcal{C}(S)$ intersects $Y$ essentially, we define $\pi_{Y}(\gamma)$ to be the collection of curves in $\mathcal{C}(Y)$ obtained by resolving the essential arcs of $\gamma \cap Y$ into simple closed curves. More precisely, if $X$ is a finite area hyperbolic structure on $\operatorname{int}(S)$ chosen for reference, one can consider the arcs of intersection of the geodesic representative $\gamma^{*}$ of $\gamma$ with the realization $Y^{*}$ of $Y$ as a subsurface of $X$ with geodesic boundary. For each arc $\alpha$ of $\gamma^{*} \cap Y^{*}$, denote by $\alpha^{*}$ the shortest representative of $\alpha$ modulo the boundary of $Y^{*}$ (ie up to homotopy with endpoints constrained to $\partial Y^{*}$ ). A regular neighborhood of $\alpha^{*} \cup \partial Y^{*}$ will have boundary consisting of simple closed curves in $Y$, some of which will be nonperipheral in $Y$. The union of these nonperipheral ones makes up the collection $\pi_{Y}(\gamma) \subset \mathcal{C}(Y)$. If this collection is empty, then $\pi_{Y}(\gamma)$ is defined to be the empty set.

If $\pi_{Y}(\gamma)$ is nonempty, it is easy to see that it has uniformly bounded diameter in $\mathcal{C}(Y)$. The projection $\pi_{Y}$ is defined in exactly the same manner for pants decompositions $P$ : resolve arcs of intersection of the geodesic representatives of $P$ with the geodesic representative of $Y$ into simple closed curves in $Y$ and record those that are nonperipheral. Once again, it is easy to see that $\pi_{Y}(P)$ is a subset of $\mathcal{C}(Y)$ of uniformly bounded diameter.

We note for any nonannular subsurface $Y$, at least one curve in a pants decomposition $P$ must have essential intersection with $Y$, so one always has $\pi_{Y}(P) \neq \varnothing$. For two pairs of pants $P_{1}, P_{2}$ we will use the notation $d_{Y}\left(P_{1}, P_{2}\right)$ to denote

$$
\operatorname{diam}_{\mathcal{C}(Y)}\left(\pi_{Y}\left(P_{1}\right) \cup \pi_{Y}\left(P_{2}\right)\right) .
$$

By a result of Masur and Minsky [19], the curve complex $\mathcal{C}(Y)$ is $\delta$-hyperbolic for some $\delta$.

Let $g_{Y}$ be a geodesic in $\mathcal{C}(Y)$.

Definition 7 For any pants $P$ decomposition, denote by $\pi_{g_{Y}}(P)$ a nearest point on the geodesic $g_{Y}$ of the projection $\pi_{Y}(P)$.

It follows from the hyperbolicity of $\mathcal{C}(Y)$ that this map is coarsely well defined and that there is a constant $k \geq 1$ such that it is $k$-Lipschitz. 
Definition 8 The domain $Y_{1}$ is nested in the domain $Y_{2}$ if $Y_{1} \subset Y_{2}$. The domains $Y_{1}$ and $Y_{2}$ intersect transversely if they intersect and are not nested.

Hierarchy paths We will use a construction in [20] of a class of quasi-geodesics in $P(S)$ which we call hierarchy paths and which have the following properties. Given a positive function $f(x)$ and a real number $M>0$, let

$$
[f(x)]_{M}= \begin{cases}f(x) & \text { if } f(x) \geq M, \\ 0 & \text { otherwise. }\end{cases}
$$

Definition 9 (Hierarchy paths) Any two pants decompositions $P_{1}$ and $P_{2}$ can be connected by at least one hierarchy path $\rho=\rho\left(P_{1}, P_{2}\right):[0, n] \rightarrow P(S)$, with $\rho(0)=P_{1}$ and $\rho(n)=P_{2}$.

These paths have the following properties.

(1) There is a constant $M_{2}$ such that if $Y$ is a subsurface of $S$ with $\zeta(Y) \geq 1$ and $d_{Y}\left(P_{1}, P_{2}\right) \geq M_{2}$ then there is a maximal connected interval of times $I_{Y}=\left[t_{1}, t_{2}\right]$ such that for all $t \in I_{Y}, \partial Y$ is a curve in $\rho(t)$. We will call such a subsurface $Y$ a component domain of $\rho$. By convention, the full surface $S$ is also a component domain.

(2) For each component domain $Y$, there is a geodesic $g_{Y}(s)$ in the curve complex $\mathcal{C}(Y)$, for $s$ in a parameter interval $J_{Y}$, such that for each $t \in I_{Y}, \rho(t)$ contains a curve in $g_{Y}(s)$. Furthermore, the assignment $t \rightarrow s(t)$ is a monotonic function from $I_{Y}$ to $J_{Y}$.

(3) If $Y_{1}$ and $Y_{2}$ are component domains that intersect transversely, then there is a notion $\prec_{t}$ of time order of the two domains which is the same for any hierarchy path joining $P_{1}$ and $P_{2}$. Time ordering has the property that there is a constant $M_{1} \geq M_{2}$ such that if $Y_{1} \prec_{t} Y_{2}$ then $d_{Y_{2}}\left(P_{1}, \partial Y_{1}\right) \leq M_{1}$ and $d_{Y_{1}}\left(P_{2}, \partial Y_{2}\right) \leq M_{1}$.

(4) For any component domain $Y$, if $I_{Y}=\left[t_{1}, t_{2}\right]$, then

$$
d_{Y}\left(\rho(t), \rho\left(t_{1}\right)\right) \leq M_{1}, \quad \text { if } t \leq t_{1},
$$

and

$$
d_{Y}\left(\rho(t), \rho\left(t_{2}\right)\right) \leq M_{1}, \quad \text { if } t \geq t_{2} .
$$

(5) There exist $K^{\prime}$ and $C$ depending on $M_{1}$ so that

$$
d\left(P_{1}, P_{2}\right) \asymp K^{\prime}, C \sum_{\substack{Y \subset S \\ \text { nonannular }}}\left[d_{Y}\left(P_{1}, P_{2}\right)\right]_{M},
$$

where $\asymp K^{\prime}, C$ denotes equality up to the multiplicative factor $K^{\prime}$ and additive constant $C$. 
In [20] it is shown that $\prec_{t}$ defines a partial ordering on the component domains of a hierarchy path. The notion that two properly intersecting domains $Y_{1}$ and $Y_{2}$ are time-ordered refers to the fact that changes of projections to a pair of time ordered component domains do not occur simultaneously along a path but rather sequentially. We will denote by $\rho\left(P, P^{\prime}\right)$ a choice of a hierarchy path joining $P$ to $P^{\prime}$ in $P(S)$.

Lemma 1 (Time order) Suppose $Y_{1}$ and $Y_{2}$ are transversely intersecting domains, and $P_{1}, P_{2}$ are pants decompositions in $P(S)$ that satisfy

$$
d_{Y_{1}}\left(P_{1}, P_{2}\right) \geq 2 M_{1} \text { and } d_{Y_{2}}\left(P_{1}, P_{2}\right) \geq 2 M_{1} .
$$

Suppose $Y_{1} \prec_{t} Y_{2}$ in $\rho\left(P_{1}, P_{2}\right)$. If $R$ is another pants decomposition that satisfies

then

$$
d_{Y_{1}}\left(R, P_{2}\right)>2 M_{1},
$$

and

$$
d_{Y_{2}}\left(P_{1}, R\right) \leq 2 M_{1}
$$$$
\left|d_{Y_{2}}\left(P_{1}, P_{2}\right)-d_{Y_{2}}\left(R, P_{2}\right)\right| \leq 2 M_{1},
$$

and $Y_{1} \prec_{t} Y_{2}$ in any hierarchy path $\rho\left(R, P_{2}\right)$.

Proof Since $Y_{1} \prec_{t} Y_{2}$ in a hierarchy path $\rho\left(P_{1}, P_{2}\right)$ we have

$$
d_{Y_{1}}\left(\partial Y_{2}, P_{2}\right) \leq M_{1} \text {. }
$$

Since $2 M_{1}>M_{2}$, the subsurface $Y_{1}$ is a component domain of a hierarchy path from $R$ to $P_{2}$. Arguing by contradiction suppose $Y_{2}$ is not a component domain of this latter path, or if it is, suppose $Y_{2} \prec_{t} Y_{1}$. By the fourth property of hierarchies we would have

$$
d_{Y_{1}}\left(R, \partial Y_{2}\right) \leq M_{1}
$$

The triangle inequality then gives

$$
d_{Y_{1}}\left(R, P_{2}\right) \leq 2 M_{1},
$$

a contradiction to the assumption.

Thus $Y_{2}$ is a component domain in a hierarchy path $\rho\left(R, P_{2}\right)$ and we have $Y_{1} \prec_{t} Y_{2}$. Thus by the third property of hierarchies, $d_{Y_{2}}\left(\partial Y_{1}, R\right) \leq M_{1}$. Since $Y_{1} \prec_{t} Y_{2}$ in $\rho\left(P_{1}, P_{2}\right) d_{Y_{2}}\left(\partial Y_{1}, P_{1}\right) \leq M_{1}$ as well. The triangle inequality gives the first bound. The third property of hierarchies gives

and

$$
\begin{aligned}
\left|d_{Y_{2}}\left(\partial Y_{1}, P_{2}\right)-d_{Y_{2}}\left(R, P_{2}\right)\right| & \leq M_{1} \\
\left|d_{Y_{2}}\left(\partial Y_{1}, P_{2}\right)-d_{Y_{2}}\left(P_{1}, P_{2}\right)\right| & \leq M_{1} .
\end{aligned}
$$

The two inequalities together finish the proof. 


\section{Relative stability of quasi-geodesics}

We now specialize to the case when $S=S_{g, n}$ is a surface of genus $g$ with $n$ boundary components, and $\zeta(S)=3$ (in other words, $(g, n)$ is $(2,0),(1,3)$, or $(0,6))$.

Definition 10 Let $S=S_{g, n}$ where $\zeta(S)=3$. Then we say an essential subsurface $W$ of $S$ is a separated domain if $W$ is a four holed sphere or one-holed torus, and there is another four-holed sphere or one-holed torus $W^{c}$ so that $W$ and $W^{c}$ may be embedded disjointly in $S$.

Given a hierarchy path $\rho$, we denote by $X(\rho)$ the union of the image of $\rho$ and the Farey-graph products $X_{\gamma}=C(W) \times C\left(W^{c}\right)$ where the separating curve $\gamma \in \mathcal{C}(S)$ is the common boundary of the separated domains $W$ and $W^{c}$ for which either $W$ or $W^{c}$ is a component domain of $\rho$.

Now Theorem 2 is a consequence of the following result, which guarantees the existence of a contracting projection map from $P(S)$ to $X(\rho)$.

We remind the reader of an important contraction property that always exists for projections to quasi-convex subsets of $\delta$-hyperbolic metric spaces.

Definition 11 A map $\Pi$ has a $(b, c)$-contraction property if there exists $b, c>0$ such that if $d(P, Q)<b d(\Pi(P), P)$ then $d(\Pi(P), \Pi(Q))<c$.

In particular, if $X$ is a $\delta$-hyperbolic metric space then there is a pair $(b, c)$ so that if $g$ is any geodesic lying in $X$ then the nearest point projection $\pi_{g}: X \rightarrow g$ satisfies a $(b, c)$-contraction property. If a map $\Pi$ satisfies a $(b, c)$-contraction property, for some $b, c$, we say it has the contraction property.

Theorem 12 Fix a hierarchy path $\rho=\rho\left(P_{1}, P_{2}\right)$. There exists a projection map

$$
\Pi: P(S) \rightarrow X(\rho)
$$

that is coarsely idempotent, coarsely Lipschitz and has the contraction property.

Moreover the projection $\Pi$ satisfies the following conditions: there is a constant $K$ depending only on the topology of $S$ so that for each $P \in P(S)$ we have:

(1) Each nonseparated component domain $Z$ of $\rho$ satisfies

$$
d_{Z}\left(\Pi(P), \pi_{g_{Z}}(P)\right)<K \text {. }
$$


(2) Each separated component domain $W$ and its complement $W^{c}$ satisfy

$$
d_{W}\left(\Pi(P), \pi_{W}(P)\right)<K, \quad d_{W^{c}}\left(\Pi(P), \pi_{W^{c}}(P)\right)<K .
$$

We refer to the final two conditions of the theorem as the relative synchronization property for the map $\Pi$. Notice that in condition (2) we do not require that the projection $\Pi(P)$ be close to any geodesic in $\mathcal{C}(W)$ or $\mathcal{C}\left(W^{c}\right)$.

Proof We break the proof into three parts. In part (i), we construct the projection. In part (ii), we verify that $\Pi$ is relatively synchronized, coarsely idempotent and coarsely Lipschitz. Finally, in part (iii) we show the projection $\Pi$ satisfies the contraction property.

Part (i): Constructing the projection Let $\rho:[0, n] \rightarrow P(S)$ be a hierarchy path with $\rho(0)=P_{1}$ and $\rho(n)=P_{2}$. Let

$$
D(\rho)=S \cup\left\{Y: d_{Y}\left(P_{1}, P_{2}\right) \geq 2 M_{1}\right\} .
$$

Given $P$ in $P(S)$, let

$$
D(P, \rho)=S \cup\left\{Y: \min \left(d_{Y}\left(P_{1}, P\right), d_{Y}\left(P, P_{2}\right)\right) \geq 2 M_{1}\right\} .
$$

First assume $D(P, \rho)$ contains a separated domain $W$, and either $W$ or $W^{c}$ is in $D(\rho)$. It follows immediately from Lemma 1 that there cannot be any other separated domain (other than $W^{c}$ ) with the same property. In this case set

$$
\Pi(P)=\partial W \cup \pi_{W}(P) \cup \pi_{W^{c}}(P)
$$

in the Farey-graph product $X_{\partial W}$.

Now assume there is no such separating domain. Again from Lemma 1 two subsurfaces $Y_{1}, Y_{2}$ in $D(P, \rho) \cap D(\rho)$ cannot transversely intersect. Then we let $B(P, \rho)$ denote the union of all the isotopy classes of boundary components of the subsurfaces in $D(P, \rho) \cap D(\rho)$ that are nonperipheral in $S$. We note that $B(P, \rho)$ may be empty. If it is nonempty it is a simplex in $\mathcal{C}(S)$. If $B^{*}(P, \rho)$ denotes the realization of these isotopy classes as a disjoint union of simple closed curves on $S$ then consider the complement $\left.S \backslash N\left(B^{*}(P, \rho)\right)\right)$ of a union of annular neighborhoods of these curves. If this complement contains a subsurface $Y$ that is not a 3 -holed sphere, then $Y$ is unique, since it cannot be a separated domain by assumption, and furthermore $Y$ lies in $D(P, \rho) \cap D(\rho)$. In this case $Y$ is minimal in $D(P, \rho) \cap D(\rho)$ with respect to the order induced by inclusion (we remind the reader that $Y$ may be the full surface $S$ ).

Recall $k$ is the Lipschitz constant for the projection $\pi_{g_{W}}$. Denote by $\mathcal{W}(P, \rho)$ the (possibly empty) set of all $W \subset Y$ such that: 
- $d_{W}\left(P_{1}, P_{2}\right)>10 k M_{1}$.

- $I_{W} \cap I_{Y} \neq \varnothing$.

Note in particular that the first condition implies $W \in D(\rho)$. Since $Y$ is minimal in $D(P, \rho)$, we have $W \notin D(P, \rho)$ so $d_{W}\left(P, P_{i}\right) \leq 2 M_{1}$ for either $P_{1}$ or $P_{2}$. Then we claim that the following order relationship holds. Either

(1) $d_{W}\left(P_{1}, \pi_{g_{W}}(P)\right) \leq 5 k M_{1}$ and we say $P<W$, or

(2) $d_{W}\left(\pi_{g_{W}}(P), P_{2}\right) \leq 5 k M_{1}$ and we say $P>W$.

(The definition of $\mathcal{W}(P, \rho)$ and the triangle inequality says that both inequalities cannot hold). To prove the claim assume without loss of generality that $d_{W}\left(P, P_{1}\right) \leq 2 M_{1}$. Let $v_{0}$ be the initial vertex of the geodesic $g_{W}$. By property (4) of hierarchy paths, we have $d_{W}\left(P_{1}, v_{0}\right) \leq M_{1}$, and so by the triangle inequality,

$$
d_{W}\left(P, v_{0}\right) \leq 3 M_{1} .
$$

Since $\pi_{g_{W}}(P)$ is the closest vertex in $g_{W}$ we then have

$$
d_{W}\left(P, \pi_{g_{W}}(P)\right) \leq 3 M_{1}
$$

and so again by the triangle inequality

$$
d_{W}\left(P_{1}, \pi_{g_{W}}(P)\right) \leq 5 M_{1} \leq 5 k M_{1}
$$

proving the claim.

Let $\mathcal{W}_{-}$denote the set of $W \in \mathcal{W}(P, \rho)$ with $P>W$ and let $\mathcal{W}_{+}$denote the set of $W \in \mathcal{W}(P, \rho)$ with $P<W$.

Define the subsets of the parameter values

$$
U_{-}=\bigcup_{W \in \mathcal{W}_{-}} I_{W} \text { and } U_{+}=\bigcup_{W \in \mathcal{W}_{+}} I_{W}
$$

Then we let $i_{1}^{\prime}$ be the maximum parameter value in $U_{-}$and $i_{2}^{\prime}$ be the minimum parameter value in $U_{+}$. (If $U_{-}=\varnothing$ take $i_{1}^{\prime}$ to be the initial point of $I_{Y}$ and similarly if $U_{+}=\varnothing$ take $i_{2}^{\prime}$ the maximal value of $I_{Y}$ ). Let

$$
P_{1}^{\prime}=\rho\left(i_{1}^{\prime}\right) \text { and } P_{2}^{\prime}=\rho\left(i_{2}^{\prime}\right) .
$$

There is a connected set $J$ of vertices on $g_{Y}$ that are vertices of pants decompositions between $P_{1}^{\prime}=\rho\left(i_{1}^{\prime}\right)$ and $P_{2}^{\prime}=\rho\left(i_{2}^{\prime}\right)$. By the hyperbolicity of $\mathcal{C}(Y)$ there are a bounded 
number of vertices in $J$ that are closest to the projection of $P$ into $\mathcal{C}(Y)$. Pick one such closest $v$. Let $\rho(j)$ be a pants decomposition that contains this $v$ and define

$$
\Pi(P)=\rho(j) .
$$

This completes the construction.

Part (ii): The map $\Pi$ is relatively synchronized, coarsely idempotent and coarsely Lipschitz We first note the following Lemma. Let $Y$ be the surface determined by the construction of $\Pi$. If $Y$ is a proper subsurface, then $\Pi(P)$ contains $\partial Y$.

Lemma 2 Suppose $Y$ is a proper subsurface. Suppose $Z$ transversely intersects $Y$, and $d_{Z}\left(P_{1}, P_{2}\right) \geq 4 M_{1}$. Then $d_{Z}(P, \Pi(P)) \leq 4 M_{1}$.

Proof If the conclusion of the lemma is false, then $Z$ is a component domain of a hierarchy $\rho(P, \Pi(P))$. Since $\Pi(P)$ contains $\partial Y$, by the fourth property of hierarchies, $d_{Y}(\partial Z, P) \leq M_{1}$. On the other hand, by the triangle inequality, for either $i=1,2$ we have $d_{Z}\left(P_{i}, \Pi(P)\right) \geq 2 M_{1}$. Without loss of generality assume this holds for $i=1$. Then again by the fourth property of hierarchies, since $\Pi(P)$ contains $\partial Y$, $d_{Y}\left(P_{1}, \partial Z\right) \leq M_{1}$ and so by the triangle inequality we have

$$
d_{Y}\left(P_{1}, P\right) \leq 2 M_{1},
$$

a contradiction to the fact that $Y \in D(P, \rho)$.

We now give the proof of relative synchronization. First assume that $Z$ is not separating. The proof breaks into cases.

Case I The subsurface $Z$ transversely intersects $Y$. If $d_{Z}\left(P_{1}, P_{2}\right) \leq 4 M_{1}$, then by property (4) of hierarchies and the fact $g_{Z}$ is a geodesic,

$$
d_{Z}\left(\Pi(P), \pi_{g_{Z}}(P)\right) \leq 5 M_{1}
$$

and we can take $K=5 M_{1}$.

If $d_{Z}\left(P_{1}, P_{2}\right) \geq 4 M_{1}$, then by Lemma 2 we have $d_{Z}(P, \Pi(P)) \leq 4 M_{1}$. But then the fact that projections to geodesics are $k$-Lipschitz says that

$$
d_{Z}\left(\pi_{g_{Z}}(\Pi(P)), \pi_{g_{Z}}(P)\right) \leq 4 k M_{1} .
$$

By property (4) of hierarchies, $d_{Z}\left(\pi_{g_{Z}}(\Pi(P)), \Pi(P)\right) \leq M_{1}$. Therefore by the triangle inequality, we have the result for $K=4 k M_{1}+M_{1}$. 
Case II $Z=Y$ If we can show that some closest point projection $v^{\prime}=\pi_{g_{Y}}(P)$ satisfies

$$
d_{Y}\left(v^{\prime}, J\right) \leq 3,
$$

then by the hyperbolicity of $\mathcal{C}(Y)$, certainly $v^{\prime}$ would be within bounded distance of $\Pi(P)$, the nearest point projection in $J$ of $P$, and we would be done. Assume this is false, and assume without loss of generality that all closest $v^{\prime}$ lie before $J$ along $g_{Y}$ at distance greater than 3 .

Let $W \in W_{-}$be the domain such that the right endpoint of $I_{W}$ coincides with the left endpoint of $J$. Let $h$ be the geodesic in $\mathcal{C}(Y)$ joining $\pi_{Y}(P)$ to $v^{\prime}$. Then we first assert that every vertex of $h$ intersects $\partial W$. If this were not true then some vertex $u$ of $h$ would be within distance 1 of $\partial W$ and therefore within distance 2 of $J$.

If $d_{S}\left(u, v^{\prime}\right) \leq 1$ then $d_{S}\left(J, v^{\prime}\right) \leq 3$, a contradiction to the assumption. If $d_{S}\left(u, v^{\prime}\right) \geq 2$, then $d_{S}(u, J) \leq d_{S}\left(u, v^{\prime}\right)$, and we have contradicted that there are no closest points in $J$. This proves the assertion that every vertex of $h$ must intersect $\partial W$. But then by the first property of hierarchy paths,

$$
d_{W}\left(P, v^{\prime}\right) \leq M_{2} .
$$

By the fourth property of hierarchies,

$$
d_{W}\left(P_{1}, v^{\prime}\right) \leq M_{1} .
$$

By the triangle inequality, $d_{W}\left(P, P_{1}\right) \leq M_{1}+M_{2} \leq 2 M_{1}$. Then the Lipschitz property of projections to geodesics implies

$$
d_{W}\left(\pi_{g_{W}}(P), \pi_{g_{W}}\left(P_{1}\right)\right) \leq 2 k M_{1}
$$

and so again by the fourth property of hierarchies and the triangle inequality,

$$
d_{W}\left(P_{1}, \pi_{g_{W}}(P)\right) \leq 2 k M_{1}+M_{1}<5 k M_{1}
$$

so $P<W$. This contradiction finishes the argument.

Case III The subsurface $Y$ is a proper subsurface of $Z$. Since $\Pi(P)$ contains $\partial Y$ it is enough to show that

$$
d_{Z}\left(\partial Y, \pi_{g_{Z}}(P)\right)<3 .
$$

Assuming otherwise, since $\pi_{g_{Z}}(P)$ is the closest point projection, every vertex on the geodesic $h$ in $\mathcal{C}(Z)$ joining $\pi_{Z}(P)$ to $\pi_{g_{Z}}(P)$ intersects $Y$. Consequently, by the first property of hierarchies, we have $d_{Y}\left(P, \pi_{g_{Z}}(P)\right) \leq M_{2}$. Since $\pi_{g_{Z}}(P)$ intersects $Y$, for either $i=1,2 d_{Y}\left(P_{i}, \pi_{g_{Z}}(P)\right) \leq M_{1}$, so for that value of $i$,

$$
d_{Y}\left(P, P_{i}\right) \leq M_{1}+M_{2} \leq 2 M_{1},
$$


a contradiction to $Y \in D(P, \rho)$.

Case IV The subsurface $Z$ is a proper subsurface of $Y$. We can assume $Z$ belongs to $\mathcal{W}$; otherwise the result is immediate. Without loss of generality assume $P>Z$ so

$$
d_{Z}\left(P_{2}, \pi_{g_{Z}}(P)\right) \leq 5 k M_{1} .
$$

Since

$$
d_{Z}\left(\Pi(P), P_{2}\right) \leq M_{1}
$$

by the fourth property of hierarchies, we are done by the triangle inequality. We have proved (1).

We now establish (2). If the separating $W$ lies in $D(P, \rho)$ and either $W$ or $W^{c}$ lies in $D(\rho)$, then $\Pi(P)$ lies in $X_{\gamma}$ where $W \subset S \backslash \gamma$, and in this case $d_{W}(P, \Pi(P))=0$. The cases that $W \notin D(P, \rho)$ or both $W, W^{c} \notin D(\rho)$ follows exactly as in (1).

It follows from relative synchronization that the map is coarsely idempotent; there is a constant $K$ such that if $P \in \rho[0, n]$ then $d(\Pi(P), P) \leq K$.

To see that the map is coarsely Lipschitz, we note that the image depends only on the projections of $P$ to subsurfaces of $S$. Since projections are Lipschitz maps, there is a constant $C$ such that if $d_{W}\left(P, P^{\prime}\right)=1$, then $d_{W}\left(\Pi(P), \Pi\left(P^{\prime}\right)\right) \leq C$. Now the Lipschitz property follows from $(2-1)$.

Part (iii): The projection $\Pi$ has the contraction property $\quad$ We begin this section by establishing further properties of the projection map $\Pi$. Let $\rho^{\prime}:[0, N] \rightarrow P(S)$ denote the hierarchy joining $P$ and $\Pi(P)$. The next lemma says that $\Pi$ is almost a nearest point projection on $\rho$.

Lemma 3 Suppose $Z$ is a component domain of $\rho^{\prime}$ with corresponding geodesic $g_{Z}^{\prime}$ and parameter interval $I_{Z}^{\prime}$. Let $j_{Z}^{\prime}$ be the last parameter value and $k_{Z}^{\prime}$ any parameter value.

- If $Z$ is also a component domain of $\rho$ with geodesic $g_{Z}$, then

$$
d_{Z}\left(\pi_{g_{Z}}\left(P_{k_{Z}^{\prime}}\right), \pi_{g_{Z}}\left(P_{j_{Z}^{\prime}}\right)\right) \leq 2 k M_{1}+K+M_{1}+2 k \delta
$$

where $\mathcal{C}(Z)$ is $\delta$ hyperbolic.

- The closest point projection $\pi_{g_{Z}^{\prime}}$ satisfies

$$
d_{Z}\left(\pi_{g_{Z}^{\prime}}(\widehat{P}), P_{j_{Z}^{\prime}}\right) \leq(4+2 k) \delta+M_{1}(3 k+3)+K+1
$$

for all $\widehat{P} \in \rho$. 
Proof Let $i_{Z}^{\prime}$ be the initial parameter value of $I_{Z}^{\prime}$. By the fourth property of hierarchies, we have

$$
d_{Z}\left(P, P_{i_{Z}^{\prime}}\right) \leq M_{1} \text { and } d_{Z}\left(\Pi(P), P_{j_{Z}^{\prime}}\right) \leq M_{1} .
$$

Since the projections to a geodesic is $k$-Lipschitz, we get (3-2) $d_{Z}\left(\pi_{g_{Z}}(P), \pi_{g_{Z}}\left(P_{i_{Z}^{\prime}}\right)\right) \leq k M_{1}$ and $d_{Z}\left(\pi_{g_{Z}}(\Pi(P)), \pi_{g_{Z}}\left(P_{j_{Z}^{\prime}}\right)\right) \leq k M_{1}$. Again applying the fourth property of hierarchies, we have

$$
d_{Z}\left(\Pi(P), \pi_{g_{Z}}(\Pi(P))\right) \leq M_{1} .
$$

Thus by relative synchronization we have

$$
d_{Z}\left(\pi_{g_{Z}}(P), \pi_{g_{Z}}(\Pi(P))\right) \leq K+M_{1} .
$$

The triangle inequality applied to inequalities (3-2) and (3-4) gives

$$
d_{Z}\left(\pi_{g_{Z}}\left(P_{i_{Z}^{\prime}}\right), \pi_{g_{Z}}\left(P_{j_{Z}^{\prime}}\right)\right) \leq 2 k M_{1}+K+M_{1} .
$$

The first conclusion which is stated for any parameter value now follows from the hyperbolicity of $\mathcal{C}(Z)$ and the fact that projections are Lipschitz.

We prove the second statement. Notice first that if $Z$ is not component domain of $\rho$, then by the first and fourth property of hierarchies and the triangle inequality,

$$
d_{Z}\left(P, P_{j_{Z}^{\prime}}\right) \leq M_{2}+M_{1}
$$

and the projection of $P$ to $g_{Z}^{\prime}$ is even closer.

Now suppose $Z$ is a component domain with geodesic $g_{Z}$. Let $v$ be any vertex of $g_{Z}$ and let $v^{\prime}=\pi_{g_{Z}^{\prime}}(v)$ its closest point projection on $g_{Z}^{\prime}$. Let $v^{\prime \prime}=\pi_{g_{Z}}\left(v^{\prime}\right)$ the closest point projection of $v^{\prime}$ on $g_{Z}$. The first conclusion of the Lemma and the fourth property of hierarchies imply that

$$
d_{Z}\left(P_{j_{Z}^{\prime}}, v^{\prime \prime}\right) \leq 2 k M_{1}+K+M_{1}+2 k \delta+2 M_{1}+1 .
$$

Since $v^{\prime \prime}$ is the closest point to $v^{\prime}$ on $g_{Z}$, the $\delta$ hyperbolicity of $\mathcal{C}(Z)$ implies that any geodesic $h$ joining $v^{\prime}$ to $v$ must pass within $2 \delta$ of $v^{\prime \prime}$ and therefore by the above bound, within $(2+2 k) \delta+M_{1}(2 k+3)+K+1$ of $P_{j_{Z}^{\prime}}$. This gives the bound for $d_{Z}\left(v^{\prime}, P_{j_{Z}^{\prime}}\right)$. Since any $\widehat{P} \in \rho$ is distance at most $M_{1}$ from $g_{Z}$ and the projections to geodesics are $k$ Lipschitz, the result now follows from the triangle inequality.

Lemma 4 There exists a constant $K^{\prime \prime}$ such that for any pants decomposition $Q^{\prime}=$ $\rho^{\prime}(i)$ in the hierarchy $\rho(P, \Pi(P))$,

$$
d\left(\Pi\left(Q^{\prime}\right), \Pi(P)\right) \leq K^{\prime} .
$$


Proof We bound $d\left(\Pi(P), \Pi\left(Q^{\prime}\right)\right)$ using the distance formula (2-1). Since $\Pi$ is Lipschitz we need only bound $d_{Z}\left(\Pi(P), \Pi\left(Q^{\prime}\right)\right)$ for those $Z$ that are component domains of both $\rho$ and the subhierarchy of $\rho^{\prime}$ joining $P$ and $Q^{\prime}$.

Now the first part of Lemma 3 says that the geodesic $g_{Z}^{\prime}$ has bounded diameter projection to $g_{Z}$. This together with the fourth property of hierarchies and the fact that projection to $g_{Z}$ is Lipschitz bounds the projection of any two points in the subhierarchy.

Now let $\Pi^{\prime}$ denote the projection to the hierarchy $\rho^{\prime}$.

Lemma 5 There is $K^{\prime \prime \prime}$ and $C$, such that if $Z$ is a subsurface and $Q$ is a pants decomposition such that

$$
d_{Z}\left(\Pi^{\prime}(Q), \Pi(P)\right) \geq C
$$

then:

(1) $d_{Z}(Q, \Pi(P)) \geq 2 M_{1}$.

(2) $d_{Z}\left(Q, \Pi(Q) \geq 2 M_{1}+K\right.$.

(3) $d_{Z}(\Pi(P), \Pi(Q)) \leq K^{\prime \prime \prime}$.

(4) For any $W$, if $d_{W}\left(Q, \Pi^{\prime}(Q)\right) \geq C+2 M_{1}$ then $d_{W}(Q, \Pi(P)) \geq 2 M_{1}$.

Proof Let $Q^{\prime}=\Pi^{\prime}(Q)$. In order to bound the distance in $\mathcal{C}(Z)$ between the projections of $\Pi(P)$ and $\Pi(Q)$, we can assume that $Z$ is a component domain of $\rho$ with geodesic $g_{Z}$. We consider the geodesic $g$ in $\mathcal{C}(Z)$ joining $\pi_{Z}(\Pi(P))$ and $\pi_{Z}(\Pi(Q))$. By the fourth property of hierarchies and the hyperbolicity of $\mathcal{C}(Z)$, there is a constant $\delta^{\prime}$ such that the geodesic $g$ is Hausdorff distance $\delta^{\prime}$ from $g_{Z}$. By relative synchronization and Lemma 3 , there is a $\delta^{\prime \prime}$ such that the closest point projection of $\pi_{Z}\left(Q^{\prime}\right)$ on $g$ is within $\delta^{\prime \prime}$ of $\pi_{Z}(\Pi(P))$ and the closest point projection of $\pi_{Z}(Q)$ on $g$ is within $\delta^{\prime \prime}$ of $\pi_{Z}(\Pi(Q))$.

We consider the quadrilateral in $\mathcal{C}(Z)$ with vertices

$$
\left\{\pi_{Z}(Q), \pi_{Z}\left(Q^{\prime}\right), \pi_{Z}(\Pi(P)), \pi_{Z}(\Pi(Q))\right\} .
$$

The hyperbolicity of $\mathcal{C}(Z)$ implies that there are constants $C$ and $\delta^{\prime \prime \prime}$ depending on $\delta^{\prime}$ and $\delta^{\prime \prime}$ so that for $d_{Z}\left(Q^{\prime}, \Pi(P)\right)>C$ we have

$$
d_{Z}(Q, \Pi(P)) \geq 2 M_{1} \text { and } d_{Z}(Q, \Pi(Q)) \geq 2 M_{1}+K,
$$

and the geodesic $h$ joining $\pi_{Z}(Q)$ and $\pi_{Z}(\Pi(Q))$ passes within $\delta^{\prime \prime \prime}$ of $\pi_{Z}(\Pi(P))$. We will choose $C$ so that

$$
C>(4+2 k) \delta+M_{1}(3 k+3)+K+1,
$$


the constant on the right side coming from Lemma 3. Taken together with the fact that closest point projection of $\pi_{Z}(Q)$ on $g$ is within $\delta^{\prime \prime}$ of $\pi_{Z}(\Pi(Q))$, we obtain the bound

$$
d_{Z}(\Pi(P), \Pi(Q))<2 \delta^{\prime \prime \prime}+\delta^{\prime \prime}
$$

by an application of the triangle inequality. Setting $K^{\prime \prime \prime}=2 \delta^{\prime \prime \prime}+\delta^{\prime \prime}$, we have the first three statements of the Lemma.

To prove the last statement, notice again by the first part that for $d_{W}\left(Q^{\prime}, \Pi(P)\right) \geq C$, we have

$$
d_{W}(Q, \Pi(P)) \geq 2 M_{1} .
$$

If $d_{W}\left(Q^{\prime}, \Pi(P)\right) \leq C$, then the assumption $d_{W}\left(Q, Q^{\prime}\right) \geq C+2 M_{1}$ gives

$$
d_{W}\left(Q, \Pi(P) \geq 2 M_{1}\right.
$$

by the triangle inequality.

Proof of Theorem 12 To conclude the proof of Theorem 12 we seek a pair $(b, c)$ so that the map $\Pi$ satisfies an $(b, c)$-contraction property. Choose $b$ so that

$$
b<\frac{1}{2 k^{\prime}}
$$

where $k^{\prime}$ is the Lipschitz constant for the map $\Pi^{\prime}$. Then since $\Pi^{\prime}(P)=P$ we have

$$
\begin{aligned}
d\left(P, \Pi^{\prime}(Q)\right) & =d\left(\Pi^{\prime}(P), \Pi^{\prime}(Q)\right) \\
& \leq k^{\prime} d(P, Q) \\
& \leq b k^{\prime} d(P, \Pi(P)) \\
& <\frac{d(P, \Pi(P))}{2} .
\end{aligned}
$$

This implies

$$
d\left(\Pi^{\prime}(Q), \Pi(P)\right)>\frac{d(P, \Pi(P))}{2} .
$$

Set $Q^{\prime}=\pi^{\prime}(Q)$. There is a constant $a_{0}$ such that if $d(P, \Pi(P)) \geq a_{0}$, the above inequality and the distance formula (2-1) guarantees the existence of a domain $Z$ for the hierarchy path $\rho^{\prime}$ such that

$$
d_{Z}\left(Q^{\prime}, \Pi(P)\right) \geq C+2 M_{1}+K
$$

where $C$ is the constant given by Lemma 5. Assume then that

$$
d(P, \Pi(P)) \geq a_{0} .
$$


Let $g_{Z^{\prime}}$ be the geodesic in $\mathcal{C}(Z)$ in $\rho^{\prime}$; recall $g_{Z^{\prime}}$ has terminal parameter value $j_{Z^{\prime}}$. Then the fourth property of hierarchies implies

$$
d_{Z}\left(Q^{\prime}, P_{j_{Z}^{\prime}}\right) \geq C+M_{1}+K .
$$

By the distance formula (2-1), bounding $d(\Pi(P), \Pi(Q))$ is equivalent to bounding $d_{W}(\Pi(P), \Pi(Q))$ for each component domain $W$ of $\rho$ and by Lemma 4 this is equivalent to bounding $d_{W}\left(\Pi(Q), \Pi\left(Q^{\prime}\right)\right)$. We first note that if

$$
d_{W}\left(Q, Q^{\prime}\right) \leq C+2 M_{1}+K
$$

then $d_{W}\left(\Pi(Q), \Pi\left(Q^{\prime}\right)\right) \leq k^{\prime}\left(C+2 M_{1}+K\right)$, since the map $\Pi$ is $k^{\prime}$-Lipschitz.

Thus we can restrict to domains $W$ with $d_{W}\left(Q, Q^{\prime}\right) \geq C+2 M_{1}+K$.

Claim 13 If $d_{W}(\Pi(P), \Pi(Q)) \geq 2 M_{1}+2 M_{2}$, ie $W \in D(\rho)$, then $Z \subseteq W$.

By way of contradiction first assume $W$ transversely intersects $Z$. By the last conclusion of Lemma 5 we have

and

$$
\begin{aligned}
& d_{W}(Q, \Pi(P)) \geq 2 M_{1} \\
& d_{Z}(Q, \Pi(P)) \geq 2 M_{1} .
\end{aligned}
$$

As $W$ and $Z$ transversely intersect, they are time ordered in any hierarchy $\rho(Q, \Pi(P))$. If $Z \prec_{t} W$, then since $d_{Z}\left(Q^{\prime}, \Pi(P)\right) \geq 2 M_{1}$, an application of Lemma 1 with $Q^{\prime}=R$ from that lemma says that $d_{W}\left(Q, Q^{\prime}\right) \leq 2 M_{1}$, a contradiction.

Thus $W \prec_{t} Z$ in $\rho(Q, \Pi(P))$. We apply Lemma 1 again using the hierarchy $\rho(Q, \Pi(P))$ this time with $R=\Pi(Q)$. Since $d_{Z}(Q, \Pi(Q)) \geq 2 M_{1}$ by Lemma 5 , we conclude that $d_{W}(\Pi(P), \Pi(Q)) \leq 2 M_{1}$, a contradiction. We have ruled out $W$ transversely intersecting $Z$.

Next suppose $W \subsetneq Z$. By the $K$ relative synchronization of the projection $\Pi^{\prime}$, we have

$$
d_{Z}\left(Q^{\prime}, \pi_{g_{Z}^{\prime}}(\partial W)\right) \leq K
$$

so by (3-5) and the triangle inequality,

$$
d_{Z}\left(\pi_{g_{Z}^{\prime}}(\partial W), P_{j_{Z}^{\prime}}\right) \geq C+M_{1} .
$$

On the other hand, since $W$ is assumed to be a component domain of $\rho$, by the second conclusion of Lemma 3

$$
d_{Z}\left(\pi_{g_{Z}^{\prime}}(\partial W), P_{j_{Z}^{\prime}}\right) \leq(4+2 k) \delta+2 M_{1}(2 k+1)+M_{2}+K<C
$$

and again we have a contradiction. This proves the claim. 
By the claim then we need only bound $d_{W}\left(\Pi(Q), \Pi\left(Q^{\prime}\right)\right)$ for $Z \subseteq W$. By third conclusion of Lemma 5 we have the bound for $W=Z$. The remaining possibility is $W$ contains $Z$. By relative synchronization,

$$
d_{W}\left(\Pi(P), \pi_{g_{W}}(\partial Z)\right) \leq K, \quad d_{W}\left(\Pi(Q), \pi_{g_{W}}(\partial Z)\right) \leq K .
$$

The desired bound $\left.d_{W}(\Pi(P), \Pi(Q))\right) \leq 2 K$ is given by the triangle inequality.

Finally we consider the possibility that $d(P, \Pi(P)) \leq a_{0}$ so that $d(P, Q) \leq a_{0} b$ by assumption. Then since $\Pi$ is $\hat{k}$ Lipschitz for some $\hat{k}$ we get a bound

$$
d(\Pi(P), \Pi(Q)) \leq \hat{k} a_{0} b
$$

in this case. Taking $c=\max \left(2 K, \hat{a}_{0} b\right)$ we get the desired bound.

Proof of Theorem 2 This follows from the usual Mostow type argument. A proof in this context is given by Lemma 7.1 of [19]: by Theorem 12 a quasi-geodesic cannot stray far from the set $X(\rho)$ due to inefficiency outside of a bounded neighborhood of the projection image.

The notion of strong relative hyperbolicity with respect to a collection of subsets, introduced for groups by Farb [13] (see Brock and Farb [7] for a metric space notion in a similar context to this paper) finds its currently accepted form in $[11 ; 10]$.

Definition 14 A metric space is said to be strongly relatively hyperbolic with respect to a collection of subsets $\mathcal{H}$ if:

(1) Given $K$ there exists $M$ such that the intersection of $K$ neighborhoods of any two subsets from $\mathcal{H}$ is $M$ bounded.

(2) Given $L, C$, there is $M$ such that for any pair of points $x, y$ and subset $A$ from the collection $\mathcal{H}$, if $d(x, y) \geq 3 \max (d(x, A), d(y, A))$, then any $(L, C)$ quasi-geodesic between $x, y$ crosses the $M$ neighborhood of $A$.

(3) For every $k$, there exists $M$ such that every thick $k$-gon belongs to the $M$ neighborhood of one of the sets in $\mathcal{H}$.

By work of Druţu [10], the last condition can be replaced by:

(3*) For positive constants $L$ and $C$ there are constants $M$ and $M^{\prime}$, such that for any $(L, C)$-quasi-geodesic triangle in $X$, there exists a set $A$ in the collection $\mathcal{H}$ whose $M$ neighborhood intersects the three sides of the triangle, such that the pairs of entrance points of the sides in this neighborhood starting from the same vertex are distance at most $M^{\prime}$ apart. 
The proof of Theorem 1 hinges on the following Proposition, verifying a condition reminiscent of Farb's bounded coset penetration property of $[13 ; 7]$.

Proposition 1 Given $K, C$ there are $K_{1}, K_{2}$ with the property that if $\phi$ is any $(K, C)-$ quasi-geodesic joining pants decompositions, $P_{1}$ and $P_{2}$, and $\rho=\rho\left(P_{1}, P_{2}\right)$ is a hierarchy joining $P_{1}, P_{2}$, then for any for any Farey-graph product $X_{\gamma} \subset X(\rho)$, the path $\phi$ and the hierarchy $\rho$ enter and exit a $K_{1}$ neighborhood of $X_{\gamma}$ within distance $K_{2}$ of each other.

Proof By Theorem 2, given $(K, C)$, there exists $K^{\prime}$ such that any $(K, C)$-quasigeodesic $\phi(n)$ stays within $K^{\prime}$ of $X(\rho)$ where $\rho=\rho\left(P_{1}, P_{2}\right)$. By relative synchronization and the distance formula (2-1) there is a constant $K_{1}$ depending on $K^{\prime}$ such that for all $n$,

$$
d(\phi(n), \Pi(\phi(n))) \leq K_{1} .
$$

Now given a Farey-graph product $X_{\gamma} \subset X(\rho)$ with complementary domains $X \backslash \gamma=$ $W \cup W^{c}$, let $n$ be the maximal parameter value such that for all $j=1, \ldots, n, \phi(j)$ does not lie in $\mathcal{N}_{K_{1}}\left(X_{\gamma}\right)$, the $K_{1}$ neighborhood of the product $X_{\gamma}$.

Let $I_{W}, I_{W^{c}}$ the parameter intervals for $W, W^{c}$. (at least one of which is nonempty). Now let $j \leq n$. If

$$
\max \left(\left|I_{W}\right|,\left|I_{W^{c}}\right|\right) \geq k,
$$

we claim that either $\Pi(\phi(j)) \in X_{\gamma^{\prime}}$ where some complementary component of $\gamma^{\prime}$ is time ordered before $W$ or $W^{c}$, or $\Pi(\phi(j))=\rho\left(j^{\prime}\right)$ where $j^{\prime}$ satisfies

$$
j^{\prime}<I_{W} \cup I_{W^{c}} .
$$

Suppose on the contrary, there exists $j_{1}, j_{2} \leq n$ such that

$$
\Pi\left(\phi\left(j_{1}\right)\right) \in X_{\gamma_{1}} \cup \rho\left(j^{\prime}\right)
$$

for some complementary component of $\gamma_{1}$ time ordered before $W$ or $W^{c}$ and $j^{\prime}<$ $I_{W} \cup I_{W^{c}}$ and

$$
\Pi\left(\phi\left(j_{2}\right)\right) \in X_{\gamma_{2}} \cup \rho\left(j^{\prime \prime}\right)
$$

for some complementary component of $\gamma_{2}$ time ordered after $W$ or $W^{c}$ and $j^{\prime \prime}>$ $I_{W} \cup I_{W^{c}}$.

Since $X_{\gamma}$ separates $X(\rho)$ and $\Pi\left(\phi\left(j_{1}\right)\right)$ and $\Pi\left(\phi\left(j_{2}\right)\right)$ lie in distinct components of $X(\rho) \backslash X_{\gamma}$, any path joining $\Pi\left(\phi\left(j_{1}\right)\right)$ and $\Pi\left(\phi\left(j_{2}\right)\right)$ must enter $X_{\gamma}$. Since the map $\Pi$ is $k$-Lipschitz and $\max \left(\left|I_{W}\right|,\left|I_{W^{c}}\right|\right) \geq k$, there must be some $j_{1}<j_{0}<j_{2}$ such that $\Pi\left(\phi\left(j_{0}\right)\right) \in X_{\gamma}$, contrary to assumption, proving the claim. 
Then since $d(\Pi(\phi(j)), \phi(j)) \leq K_{1}$ it follows from (2-1) and the fourth property of hierarchies, that for all $j \leq n$,

$$
d_{W}\left(\phi(j), P_{1}\right) \leq K_{1}+M_{1} \text { and } d_{W^{c}}\left(\phi(j), P_{1}\right) \leq K_{1}+M_{1} .
$$

This implies that the first time $\phi$ enters a $K_{1}$ neighborhood of $X_{\gamma}$ (at time $n+1$ ) there is a bound on $d_{W}\left(P_{1}, \phi(n+1)\right)$ and $d_{W^{c}}\left(P_{1}, \phi(n+1)\right)$ and this in turn, by the fourth property of hierarchies implies that $\phi$ enters this neighborhood a bounded distance $K_{2}$ from where $\rho$ does. The same is true for the minimal parameter value $m$ where $\phi(j) \notin \mathcal{N}_{K_{1}}\left(X_{\gamma}\right)$ for all $j \geq m$.

For those $X_{\gamma}$ such that $\max \left(\left|I_{W}\right|,\left|I_{W^{c}}\right|\right) \leq k$, note that the bound $d(\phi(n), \Pi(\phi(n))) \leq$ $K_{1}$ bounds

$$
\max \left(d_{W}\left(\phi(n), P_{i}\right), d_{W^{c}}\left(\phi(n), P_{i}\right)\right) .
$$

In particular, $\phi(n)$ enters any neighborhood of $X_{\gamma}$ a uniformly bounded distance $K_{2}$ away from where $\rho(n)$ does. This completes the proof.

Proof of Theorem 1 We show property (1) is satisfied. Let $X_{\gamma}, X_{\gamma^{\prime}}$ a pair of Fareygraph products. Let $W$ and $W^{c}$ the separating domains with boundary $\gamma$. Since $\gamma^{\prime}$ intersects both $W$ and $W^{c}$, and every curve in the Farey-graph product $X_{\gamma^{\prime}}$ is disjoint from $\gamma^{\prime}$, the projection of the entire Farey-graph product $X_{\gamma^{\prime}}$ to $W$ and $W^{c}$ lies at bounded distance from the projection of $\gamma^{\prime}$. Since the projection map is Lipschitz, the same is true of a $K$ neighborhood of $X_{\gamma^{\prime}}$. This together with the distance formula (2-1) gives the first condition.

To verify (2), we can assume

$$
d(x, y) \geq 6 K^{\prime}\left(4 M_{1}+M_{2}\right)+3 K^{\prime} C .
$$

Again let $W$ and $W^{c}$ be the components of the complement of $\gamma$. Let $a \in X_{\gamma}$ the closest point to $x$ and $b \in X_{\gamma}$ the closest point to $y$. By the fourth property of hierarchies,

$$
d_{W}(x, a) \leq M_{1}, \quad d_{W^{c}}(x, a) \leq M_{1}
$$

with the same inequalities with $y$ and $b$. Then we have

$$
\begin{aligned}
d_{W}(x, y)+d_{W^{c}}(x, y) & \geq d_{W}(a, b)+d_{W^{c}}(a, b)-4 M_{1} \\
& \geq \frac{d(a, b)}{K^{\prime}}-C-4 M_{1} \\
& \geq \frac{d(x, y)}{3 K^{\prime}}-C-4 M_{1} \\
& \geq 2 M_{2} .
\end{aligned}
$$


Then by the first property of hierarchies, any hierarchy joining $x$ and $y$ passes through $X_{\gamma}$, and therefore by Proposition 1 any $(L, C)$-quasi-geodesic passes through a bounded neighborhood of $X_{\gamma}$.

To verify $\left(3^{*}\right)$, without loss of generality assume $d_{W}(x, y) \geq M_{2}$ for some separated domain $W$. Then any hierarchy joining $x$ and $y$ passes through the corresponding Farey-graph product $X_{\gamma}$ at a point $a$ that satisfies $d_{W}(x, a) \leq M_{1}$ and $d_{W^{c}}(x, a) \leq M_{1}$. If $d_{W}(x, z) \geq M_{2}$ or $d_{W^{c}}(x, z) \geq M_{2}$ the same estimates hold for the entry point $b$ for any hierarchy joining $x$ and $z$. The triangle inequality then bounds the distance between $a$ and $b$ in these domains. If a hierarchy joining $x$ and $z$ does not enter the Farey-graph product, one has the bound $M_{2}$ on $d_{W}(x, z)$ and $d_{W^{c}}(x, z)$. Thus in either case we have a bound on the distance between $a$ and $b$ in a fixed neighborhood of $X_{\gamma}$. Again by Proposition 1 this is true for any $(L, C)$-quasi-geodesic.

Proof of Theorem 3 Suppose $\phi: \mathbb{R}^{2} \rightarrow P(S)$ is a $\left(K_{0}, C_{0}\right)$-quasi-isometric embedding. For each $R>0$ join $\phi(-R, 0)$ to $\phi(R, 0)$ by a hierarchy path $\rho_{R}$. Now consider for $\left|R_{1}\right| \leq R$, the $\sqrt{2}$ quasi-geodesic $\sigma_{R, R_{1}}$ in $\mathbb{R}^{2}$ consisting of segments joining $(-R, 0)$ to $\left(-R, R_{1}\right),\left(-R, R_{1}\right)$ to $\left(R, R_{1}\right)$ and $\left(R, R_{1}\right)$ to $(R, 0)$.

Its image $\phi\left(\sigma_{R, R_{1}}\right)$ is a $K_{0} \sqrt{2}+C_{0}$ quasi-geodesic joining $\phi(-R, 0)$ and $\phi(R, 0)$. Since $\phi$ is a $\left(K_{0}, C_{0}\right)$-quasi-isometry, except for an initial and final segment on each of length $R^{\prime}$

$$
d\left(\phi\left(\sigma_{R, R_{1}}\right), \rho_{R}\right)>\frac{R^{\prime}}{K_{0}}-C_{0} .
$$

Since $\phi\left(\sigma_{R, R_{1}}\right)$ must remain a bounded distance from $X\left(\rho_{R}\right)$ it follows that for $R^{\prime} / K_{0}-C_{0}$ sufficiently large, by Theorem 1 these points on $\phi\left(\sigma_{R, R_{1}}\right)$ must lie in a bounded neighborhood of the product of Farey graphs $\left\{X_{\gamma} \mid \gamma \in \rho_{R}\right\}$.

For $R^{\prime}$ chosen sufficiently large, but fixed, the fact that bounded neighborhoods of Farey-graph products have bounded intersection guarantees that this subset of $\phi\left(\sigma_{R, R_{1}}\right)$ must lie within a bounded neighborhood of a single $X_{\gamma}$, and in addition, $\phi\left(\sigma_{R, R_{1}}\right)$ must enter and exit this neighborhood a bounded distance from where $\rho_{R}$ enters and exits the neighborhood. The above statement is true for arbitrary $R$, and therefore by enlarging $R$ while keeping $R_{1}$ fixed, we conclude there is a single $X_{\gamma}$ such that the image of the entire horizontal line $y=R_{1}$ lies in a fixed neighborhood of $X_{\gamma}$. Since this is true for arbitrary $R_{1}$ larger than a fixed size, the entire quasi-flat must lie within a bounded distance of a single $X_{\gamma}$.

Proof of Corollary 4 We claim there is no quasi-isometric embedding

$$
\varphi: \mathbb{R}^{3} \rightarrow P(S) .
$$


To see this, note that by Theorem $3 \varphi$ maps the $x-y$ plane to a bounded neighborhood of a single Farey-graph product $X_{\gamma}$ and the $y-z$ plane to a bounded neighborhood of a product $X_{\gamma^{\prime}}$. Since these planes meet along a line, we have that $X_{\gamma}$ and $X_{\gamma^{\prime}}$ have uniform neighborhoods with infinite diameter intersection, which implies that $\gamma=\gamma^{\prime}$. Composing with the nearest point projection to $X_{\gamma}$, we have a quasi-isometric embedding of $\mathbb{R}^{3}$ into a product of Farey graphs, which is impossible by [17].

\section{The boundary of the Weil-Petersson metric}

In low complexity cases, when the pants graph is Gromov hyperbolic, the collection of asymptote classes of geodesic rays is basepoint invariant and corresponds to the usual Gromov boundary of the Gromov hyperbolic space.

For a general CAT(0) space the asymptote class of an infinite geodesic ray remains a basepoint invariant notion (see Bridson and Haefliger [4]). We find that the relative stability of quasi-geodesics in $P(S)$ when $\zeta(S)=3$ provides for sufficient control over geodesic rays in the Weil-Petersson metric to give a description of the CAT $(0)$ boundary in this setting as well.

To this end, we briefly recall some standard properties of the Weil-Petersson metric and its completion. For more details, we direct the reader to Wolpert [25] and Brock [5].

The Weil-Petersson completion It is due to Wolpert and Chu that the Weil-Petersson metric is not complete. Masur examined the structure of the completion $\overline{\operatorname{Teich}(S)}$ and found a natural correspondence between the completion and the augmented Teichmüller space, consisting of marked Riemann surfaces with nodes corresponding to a pairwise disjoint collection of simple closed curves on $S$ that have been pinched.

The augmented Teichmüller space has a stratified structure organized by simplices in the curve complex. This structure is most easily described via the notion of extended Fenchel-Nielsen coordinates as follows: given a maximal simplex $\sigma$ in the curve complex $\mathcal{C}(S)$, with vertex set $\sigma^{\circ}=\left\{\alpha_{1}, \ldots, \alpha_{\zeta(S)}\right\}$ the usual associated length-twist Fenchel-Nielsen coordinates for a surface $X \in \operatorname{Teich}(S)$ are given by the product

$$
\Pi_{\alpha \in \sigma^{\circ}}\left(\ell_{\alpha}(X), \theta_{\alpha}(X)\right) \in \mathbb{R}_{+}^{\zeta(S)} \times \mathbb{R}^{\zeta(S)}
$$

indicating that $X$ is assembled from hyperbolic three-holed spheres with geodesic boundary lengths $\ell_{\alpha}(X)$ and twist parameters $\theta_{\alpha}(X), \alpha \in \sigma^{\circ}$ (see Imayoshi and Taniguchi [15]). Then the extended Fenchel-Nielsen coordinates corresponding to $\sigma$ 
parametrize the subset of $\overline{\text { Teich }(S)}$ with "nodes along $\sigma$ " by allowing the parameters $\ell_{\alpha}(X)=0$, and imposing the equivalence relation

$$
(0, \theta) \sim\left(0, \theta^{\prime}\right)
$$

for coordinates $\left(\ell_{\alpha}, \theta_{\alpha}\right)$ where $\ell_{\alpha}$ vanishes.

For any subsimplex $\eta \subset \sigma$, then, the $\eta$-stratum $\operatorname{Teich}_{\eta}(S)$ refers to the locus

$$
\left\{\ell_{\alpha}(X)=0 \Longleftrightarrow \alpha \in \eta^{\circ}\right\},
$$

in other words, the subset of $\overline{\text { Teich }(S)}$ where precisely the curves corresponding to the vertices of $\eta$ have vanishing length functions. The $\eta$-stratum has the natural structure of a (possibly empty) product of Teichmüller spaces of the complementary subsurfaces $Y \subset S$ of nonzero Teichmüller dimension in the complement $S \backslash\left\{\alpha_{1}, \ldots, \alpha_{k}\right\}$ of the simple closed curves $\left\{\alpha_{i}\right\}$ corresponding to the vertices of $\eta$.

Asymptote classes and hierarchy paths We first remark that there is a natural invariant of the asymptote class of a half-infinite hierarchy path $\rho(n), n \in \mathbb{N}$, in the pants graph, which we will call a boundary lamination. Indeed, the collection of subsurfaces $W \subset S$ for which

$$
\operatorname{diam}_{W}(\rho([0, n])) \rightarrow \infty
$$

as $n \rightarrow \infty$ form a pairwise disjoint collection of subsurfaces of $S$. Since each is a component domain for the hierarchy path $\rho(n)$, each carries a geodesic $g_{W} \subset \mathcal{C}(W)$ of infinite length. This geodesic is asymptotic to a geodesic lamination $\lambda_{W}$ (filling $W$ ) in the Gromov boundary $\partial \mathcal{C}(W)$ (see Klarreich [16]), so that for all $n$ sufficiently large, each $\rho(n)$ contains a curve in $g_{W}$.

By the distance formula $(2-1)$, if $\rho^{\prime}(n)$ is another hierarchy path for which

$$
d\left(\rho(n), \rho^{\prime}(n)\right)<D
$$

for all $n$, then $d_{W}\left(\rho(n), \rho^{\prime}(n)\right)<D^{\prime}$ for some $D^{\prime}$, from which it follows that $\rho^{\prime}(n)$ contains curves in $\mathcal{C}(W)$ asymptotic to $\lambda_{W}$. Hence the union of these $\lambda_{W}$ forms a geodesic lamination on $S$ which is an invariant of the asymptote class of $\rho(n)$.

We note that the case when $\zeta(S) \leq 2$, the asymptote class is uniquely determined by this lamination, since in these low complexity cases the boundary lamination associated to a hierarchy path is connected, and any two hierarchy paths with the same boundary lamination lie a bounded distance apart: this can be seen directly from property (4) of the definition of hierarchy paths. The distance formula (2-1) guarantees that for any proper subsurface $Y \subsetneq S$, the projections $\pi_{Y}(\rho(n))$ begin at $\pi_{Y}(\rho(0))$ and lie at a 
bounded distance from the geodesic joining $\pi_{Y}(\rho(0))$ to $\pi_{Y}(\lambda)$ (where this geodesic is infinite if $\lambda$ is a lamination in $Y$ ).

To relate this discussion to Weil-Petersson geodesics, we begin by associating to each a hierarchy path $\rho$ (via Theorem 2 ) and then associating to that the corresponding boundary lamination for the asymptote class of $\rho$ in $P(S)$.

Let $\{X(t)\}_{t=0}^{\infty}$ be a geodesic in the Weil-Petersson metric. Then by Theorem 1 of [5] the geodesic $X(t)$ describes a quasi-geodesic $\left\{P_{n}\right\}_{n=0}^{\infty}$ in $P(S)$ by taking its image under the quasi-isometry

$$
Q: \operatorname{Teich}(S) \rightarrow P(S)
$$

By Theorem 2, there is a hierarchy path $\rho(n)$, so that the quasi-geodesic $\left\{P_{n}\right\}$ stays a bounded distance from the associated set $X(\rho)$. To fix attention on the underlying pants decomposition we adopt the notation

$$
\rho(n)=Q_{n}
$$

As the ray $X(t)$ is half-infinite, the path $Q_{n}$ has the property that for some component domain $W$, we have

$$
\operatorname{diam}_{W}\left(\left\{Q_{n}\right\}_{n=0}^{\infty}\right)=\infty
$$

and, moreover, that if this property holds for more than one component domain, then the corresponding two subsurfaces are complementary separated domains.

Since each domain $W$ for $\rho$ with this property carries a unique geodesic $g_{W}$, it also carries a corresponding boundary point $\lambda$ in $\partial \mathcal{C}(W)$ to which $g_{W}$ is asymptotic. Given $\rho$ we call the union of such boundary points a boundary lamination for $\rho$. Note that such laminations are purely irrational: a boundary lamination contains no simple closed curves. When this union is disconnected, we associate real weights to each component up to scale.

The boundary laminations are topologized as follows: given a sequence $\lambda_{n}$ of boundary laminations, we say $\lambda_{n}$ converges to $\lambda$ if one of the following holds:

(1) There is a single domain $W$ and a connected $\lambda \in \partial \mathcal{C}(W)$ for which $\pi_{W}\left(\lambda_{n}\right) \rightarrow \lambda$.

(2) There are two complementary separated domains $W$ and $W^{c}$ in $S$ for which $\pi_{W}\left(\lambda_{n}\right)$ converges to $\lambda_{W} \in \partial \mathcal{C}(W)$ and $\pi_{W^{c}}\left(\lambda_{n}\right)$ converges to $\lambda_{W^{c}} \in \partial \mathcal{C}\left(W^{c}\right)$ and

$$
\lim _{n \rightarrow \infty} \frac{d_{W}\left(P, \lambda_{n}\right)}{d_{W^{c}}\left(P, \lambda_{n}\right)}=m
$$


in which case we have

$$
\begin{array}{ll}
\lambda=m \lambda_{W}+\lambda_{W^{c}} & \text { if } m \in(0, \infty), \\
\lambda=\lambda_{W} & \text { if } m=\infty, \\
\lambda=\lambda_{W^{c}} & \text { if } m=0 .
\end{array}
$$

Then we have the following.

Theorem 15 Let $S$ be a surface for which $\zeta(S) \leq 3$. Then the CAT(0) boundary of the Weil-Petersson metric is homeomorphic to the space of boundary laminations.

Proof Given a ray $r=X(t)$ in the visual sphere at $X=X(0)$, let $\lambda(r)$ denote the associated boundary lamination for $X(t)$. We first show that this association is well defined and injective.

From the discussion preceding the statement of the theorem, each infinite WeilPetersson geodesic ray $X(t)$ determines either a connected geodesic lamination $\lambda$ or a weighted sum of connected laminations $\lambda_{1}$ and $\lambda_{2}$.

In the case when we have the connected lamination $\lambda$, it is easy to see that any other geodesic ray $Y(t)$ that lies a bounded distance from $X(s(t))$ for some reparametrization $s(t)$, determines the same boundary lamination $\lambda$.

Injectivity To see that there is a unique asymptote class of geodesic rays with associated lamination $\lambda$ we assume first that the minimal subsurface $S(\lambda)=W$ containing $\lambda$ is not a separated domain.

With this assumption, the first possibility is that $W=S$. Then $\lambda$ is a filling lamination in the boundary of the curve complex of $S$. Now suppose $X_{1}(t)$ and $X_{2}(t)$ are geodesic rays through the base point $X$, each determining $\lambda$. Associated to $X_{1}(t)$ and $X_{2}(t)$ are hierarchy paths $\rho_{1}(n)$ and $\rho_{2}(n)$ each with some initial pants decomposition $Q_{0}$. The main geodesics $m_{1}$ and $m_{2}$ of $\rho_{1}$ and $\rho_{2}$ are infinite in the curve complex of $S$, and each converges to $\lambda$.

By the first property of hierarchies, for any subsurface $W$ such that $d_{W}\left(Q_{0}, \lambda\right) \geq M_{2}$, there are connected intervals of times $\left[s_{1}, t_{1}\right]$ and $\left[s_{2}, t_{2}\right]$, such that for every time in these intervals the hierarchy paths $\rho_{1}, \rho_{2}$ contain $\partial W$. Furthermore by the fourth property of hierarchies and the triangle inequality, since both hierarchies start with $Q_{0}$,

$$
d_{W}\left(\rho_{1}\left(s_{1}\right), \rho_{2}\left(s_{2}\right)\right) \leq 2 M_{1},
$$

and since both main geodesics converge to $\lambda$,

$$
d_{W}\left(\rho_{1}\left(t_{1}\right), \rho_{2}\left(t_{2}\right)\right) \leq 2 M_{1} .
$$


This means that $\rho_{1}$ and $\rho_{2}$ are bounded distance apart at these times, and the same is then true of the corresponding Weil-Petersson geodesics $X_{1}$ and $X_{2}$.

Since the Weil-Petersson metric on the completion is CAT(0), the two geodesics stay a uniform distance apart between these two points. If there are infinitely many such $W$, this proves the geodesics determine the same asymptote class. If there are only finitely many, then after some point, the hierarchy paths are bounded distance apart, since their main geodesics are bounded distance apart and projections to all subsurfaces are bounded. The same is then true for the geodesics.

Next assume $W \subsetneq S$ and $W$ is not separated. Then the boundary stratum in $\overline{\operatorname{Teich}(S)}$ determined by $\partial W$ is not a product and is in fact isometric to Teich $(W)$. Let $\sigma$ denote the simplex in the curve complex corresponding to the curves in $\partial W$ that are not in $\partial S$. The condition that $\lambda$ is a boundary lamination for $X(t)$ implies that there is a hierarchy path $\rho(n)$ whose underlying pants decomposition we again denote by $Q_{n}$, so that $\partial W$ represent curves in $Q_{n}$ for all $n$ sufficiently large. Thus $X(t)$ lies at a bounded distance from the boundary stratum $\operatorname{Teich}_{\sigma}(S)$ corresponding to the vanishing of the extended length function $\ell_{\sigma}$ for the simplex $\sigma$. Since this stratum $\operatorname{Teich}_{\sigma}(S)$ is a lower dimensional Teichmüller space $(\zeta(W)<3)$ we have that $\operatorname{Teich}_{\sigma}(S)$ is itself Gromov-hyperbolic by [7]. Thus, the lamination $\lambda$ determines a unique asymptote class of geodesics in $\operatorname{Teich}_{\sigma}(S)$, and hence in $\operatorname{Teich}(S)$.

The discussion when $W$ is a separated domain follows from the limiting case when $\lambda$ is disconnected.

When the associated boundary lamination is disconnected and breaks into components $\lambda_{1}$ and $\lambda_{2}$, then there is a separating curve $\gamma$ and a pair of complementary domains $W_{1}$ and $W_{2}$ in $S \backslash \gamma$ with $\lambda_{i} \in \partial \mathcal{C}\left(W_{i}\right)$.

In this case, the pants decompositions $Q_{n}$ contain the curve $\gamma$ for all $n$ sufficiently large. The implication for $X(t)$ is that for all $t$ sufficiently large the surface $X(t)$ has a shortest pants decomposition $P_{t}$ that is a uniformly bounded distance from a pants decomposition $Q_{n} \in X_{\gamma}$. Since the pants graph is quasi-isometric to the Weil-Petersson metric, we have that points on the geodesic $X(t)$ lie at a uniformly bounded distance from the boundary stratum $\operatorname{Teich}_{\gamma}(S)$ where the extended length function $\ell_{\gamma}$ for $\gamma$ vanishes.

The nearest point projection

$$
\wp_{\gamma}: \operatorname{Teich}(S) \rightarrow \operatorname{Teich}_{\gamma}(S)
$$

to the boundary stratum $\operatorname{Teich}_{\gamma}(S)$ determines a path

$$
Z(t)=\wp_{\gamma}(X(t))
$$


in $\operatorname{Teich}_{\gamma}(S)$. The projection of $Z(t)$ to each factor, in turn, lies a bounded distance from a unique geodesic. Let $g(t) \subset$ Teich $\left(W_{1}\right)$ denote the geodesic in the first factor and $h(t) \subset$ Teich $\left(W_{2}\right)$ the geodesic in the second.

Each infinite geodesic ray $r$ based at $g(0) \times h(0)$ in the product

$$
\left\{g(s) \times h(t) \mid s, t \in \mathbb{R}^{+}\right\}
$$

is determined by its slope, in other words, the unique value of $m \in \mathbb{R}^{+}$so that $r=\{(g(m t), h(t))\}$ where $g$ and $h$ are assumed parametrized by arclength.

Since the Weil-Petersson completion is CAT(0), it follows that $X(t)$ has a well defined slope in $\operatorname{Teich}_{\gamma}(S)$ namely, any geodesic in $\operatorname{Teich}_{\gamma}(S)$ within a uniformly bounded neighborhood of $X(t)$ has slope $m$. It follows that the boundary lamination

$$
\left[m \lambda_{1}+\lambda_{2}\right]
$$

uniquely specifies the geodesic ray $X(t)$ based at $X(0)$.

For the final case when $\lambda$ is connected but its minimal subsurface $S(\lambda)$ is a separated domain $W$, we note that this corresponds to the case above with slope $m=0$ or $m=\infty$, in other words, one of the two factors is bounded.

Surjectivity We now show that the assignment of a boundary lamination is surjective. In other words, we must show further that given any boundary lamination $\lambda$ there is a geodesic ray with that boundary lamination associated to its asymptote class.

To see this for connected $\lambda$, we take a hierarchy path $\rho(n)$ whose only infinite geodesic lies in $\mathcal{C}(S(\lambda))$ and is asymptotic to $\lambda$. We denote by $C_{n} \in \overline{\operatorname{Teich}(S)}$ the maximally noded surface obtained by pinching the curves in the underlying pants decomposition $Q_{n}$ for $\rho(n)$.

Then the sequence of Weil-Petersson geodesic rays $X_{n}(t)$ beginning at $X=X_{n}(0)$ and terminating at the maximally noded surface $C_{n}$ has a limit $X_{\infty}$ in the visual sphere based at $X$, after passing to a subsequence (by compactness of the visual sphere).

We claim $X_{\infty}(t)$ is an infinite geodesic ray. Let

$$
s_{n}=\frac{1}{\ell_{X}\left(Q_{n}\right)}
$$

where $\ell_{X}\left(Q_{n}\right)$ denotes the total length of the geodesic representatives of the curves in $Q_{n}$ on $X$. Then for each $X_{n}(t)$ we have

$$
\ell_{X_{n}(t)}\left(s_{n} Q_{n}\right) \leq 1
$$


by convexity of length functions along geodesics (see Wolpert [24]) since the length at $X$ is 1 , and the length converges to zero at the other endpoint.

Assume $X_{\infty}(t)$ has finite length $T$. Let $\mu$ be any limit of $s_{n} Q_{n}$ in $\mathcal{M L}(S)$ after passing to a subsequence. By a theorem of Klarreich [16], $\mu$ is a measure on $\lambda$.

Bicontinuity of length on $\operatorname{Teich}(S) \times \mathcal{M L}(S)$ guarantees that for any $t<T$ we have

$$
\ell_{X_{\infty}(t)}(\mu) \leq 1 .
$$

Let $\alpha$ be the (possibly empty) boundary of the minimal subsurface $S(\mu)$ containing $\mu$. Let $\sigma$ be the simplex in the curve complex corresponding so that $X_{\infty}(T) \in \operatorname{Teich}_{\sigma}(S)$. We note that

$$
\lim _{t \rightarrow T} \ell_{X_{\infty}(t)}(\mu) \leq 1
$$

which guarantees that for each simple closed curve $\gamma \in \sigma^{0}$, we have

$$
i(\gamma, \mu)=0 .
$$

Otherwise, since $\ell_{X_{\infty}(t)}(\gamma) \rightarrow 0$ as $t \rightarrow T$, we would have $\ell_{X_{\infty}(t)}(\mu) \rightarrow \infty$. (In particular, we may conclude that $\mu$ does not fill $S$ ).

Noting that $X_{n}(T)$ converges in the completion to $X_{\infty}(T)$, we have that the distance $d_{n}$ from $X_{n}(T)$ to the stratum $\operatorname{Teich}_{\sigma}(S)$ is tending to zero. Since $i(\gamma, \mu)=0$, by the choice of $Q_{n}$, for large $n$ the maximal cusps $C_{n}$ lie in the completion of the $\sigma$ stratum $\operatorname{Teich}_{\sigma}(S)$, and their distance in the completion from $X_{\infty}(T)$ is diverging.

As the $\sigma$-stratum is totally geodesic, the fact that the completion $\overline{\operatorname{Teich}(S)}$ is a $\operatorname{CAT}(0)$ space guarantees that for any $T^{\prime}>T$, the surfaces $X_{n}\left(T^{\prime}\right)$ converge into the $\sigma$-stratum as well. To see this note that the geodesics $Y_{n}(s)$ joining $X_{\infty}(T)$ to $C_{n}$ have the property that for any $s_{0}, Y_{n}\left(s_{0}\right)$ lies in the $\sigma$-stratum. But the distance of the segments $X_{n}\left(\left[T, T^{\prime}\right]\right)$ from the geodesics $Y_{n}$ tends to zero, so the distance from $X_{n}\left(T^{\prime}\right)$ to $\operatorname{Teich}_{\sigma}(S)$ tends to zero.

It follows that the finite-length geodesic segments $X_{n}\left(\left[0, T^{\prime}\right]\right)$ have endpoints converging in the completion and thus these limiting endpoints are the endpoints of a geodesic segment whose interior lies in the maximal stratum containing its endpoints (see Daskalopoulos and Wentworth [9] and Wolpert [25]).

Since one endpoint of each geodesic $X_{n}\left(\left[0, T^{\prime}\right]\right)$ is the base surface $X$, the interior of the limit segment lies in the interior of Teichmüller space. But by [25], parametrizations of the approximating geodesics $X_{n}\left(\left[0, T^{\prime}\right]\right)$ proportional to arclength converge to the parametrization proportional to arclength of the limit segment. Thus, the limit $X_{\infty}(T)$ of the sequence $\left\{X_{n}(T)\right\}$ lies in the interior of this geodesic limit, contradicting the 
assumption that $X_{\infty}(T) \in \operatorname{Teich}_{\sigma}(S)$. We conclude that the limiting geodesic $X_{\infty}$ is infinite.

It follows that we can extract a limiting infinite geodesic ray $X_{\infty}$ based at $X$, that lies within a uniform neighborhood of the union of maximal cusps $\bigcup_{n=0}^{\infty} C_{n}$. When $\lambda$ is connected it follows that $X_{\infty}$ has associated boundary lamination $\lambda$.

To treat the case when $\lambda$ is disconnected, assume that

$$
\lambda=m \lambda_{1}+\lambda_{2}
$$

where $\lambda_{i} \in \mathcal{C}\left(W_{i}\right)$ lies in the boundary of the curve complex of the separated domain $W_{i}$. Let $\gamma$ be the separating curve for which $S \backslash \gamma=W_{1} \sqcup W_{2}$. Then there is a geodesic $g_{m}$ in

$$
\operatorname{Teich}_{\gamma}(S)=\operatorname{Teich}\left(W_{1}\right) \times \operatorname{Teich}\left(W_{2}\right)
$$

of slope $m$ in the $\gamma$-stratum running through the nearest point $X^{\prime}$ to $X$ in $\operatorname{Teich}_{\gamma}(S)$. Let $C_{n} \in \overline{\operatorname{Teich}(S)}$ denote a collection of points so that $C_{0} \in \overline{\operatorname{Teich}_{\gamma}(S)}$ is the maximally noded surface closest to $X^{\prime}$, and:

(1) Each $C_{n}$ is a maximally noded surface in $\overline{\operatorname{Teich}_{\gamma}(S)}$.

(2) Each $C_{n}$ lies a uniform distance $D$ from the geodesic $g_{m}$.

(3) The pants decompositions $Q_{n}$ pinched in $C_{n}$ determine a hierarchy path $\rho(n)$ for which the projection $\pi_{W_{i}}(\rho(n))$ is asymptotic to $\lambda_{i}$ in $\partial \mathcal{C}\left(W_{i}\right)$.

Then we may apply the previous argument to conclude that the limit $X_{\infty}$ of the geodesic segments $X_{n}(t)$ joining $X$ to $C_{n}$ is an infinite geodesic ray at $X$ that lies uniformly bounded distance from the geodesic $g_{m}$; in other words, $X_{\infty}$ lies in the asymptote class with projective boundary lamination

$$
m \lambda_{1}+\lambda_{2}
$$

Continuity We now show that the assignment of a lamination to a ray is continuous. A family of infinite rays $X_{n}(t)$ based at $X(0)$ converge to $X(t)$ if there is a constant $D$ so that for each $T>0$ there is an $n$ so that

$$
d\left(X_{n}(t), X(t)\right)<D
$$

for all $t \leq T$. By the quasi-isometry between the pants graph and the Weil-Petersson metric, we have a $D^{\prime}$ for which

$$
d\left(P_{n}(t), P(t)\right)<D^{\prime}
$$


for all $t \leq T$, where $P_{n}(t)$ and $P(t)$ are shortest pants decompositions on $X_{n}(t)$ and $X(t)$ respectively (and are thus the images of $X_{n}(t)$ and $X(t)$ under the quasiisometry).

But for any $W \subset S$, the above guarantees that for any $T>0$ there is an $N_{T}$ so that

$$
d_{W}\left(P_{n}(T), P(T)\right)<D^{\prime \prime}
$$

for all $n>N_{T}$.

If $\lambda \in \partial \mathcal{C}(W)$ is a component of the boundary lamination for $X(t)$, then we have that $\pi_{W}(P(t)) \rightarrow \lambda$ as $t \rightarrow \infty$. Thus, if $\lambda_{n}$ is the boundary lamination for $X_{n}$, we have $\pi_{W}\left(\lambda_{n}\right) \rightarrow \lambda$, by an application of the fact that $\pi_{W}\left(P_{n}(T)\right)$ lies a bounded distance from the geodesic in $\mathcal{C}(W)$ joining $\pi_{W}\left(P_{n}(0)\right)$ to $\pi_{W}\left(\lambda_{n}\right)$ and that the bound (4-1) holds for each $T$ and all $n>N_{T}$.

This suffices to show continuity in the case when $\lambda$ is connected. For the disconnected case, we apply the bound (4-1) to each separated domain and observe that the divergence of the projections guarantees that the ratios converge.

To show continuity of the inverse, assume $r_{n}$ is a sequence of rays determining $\lambda_{n} ; r$ is a ray determining $\lambda$ and $\lambda_{n} \rightarrow \lambda$. We need to show $r_{n} \rightarrow r$. Since the association of laminations to infinite rays is $1-1$ and continuous on the subset of infinite rays in the visual sphere, it is enough to show that some subsequence of $r_{n}$ converges to an infinite ray $\hat{r}$. Suppose $\lambda$ fills $W$. The hierarchy $\rho_{n}$ determined by $\lambda_{n}$ gives a sequence of pants decompositions $Q_{n, k}$. If $W=S$ choose any sequence $Q_{n, k_{n}}$ where $k_{n} \rightarrow \infty$. If $W \subsetneq S$ choose a last pants decomposition $Q_{n, k_{n}}$ containing $\partial W$. In either case as in the proof of surjectivity, $Q_{n, k_{n}}$ converges projectively to a measure $\mu$ on $\lambda$. The geodesic $X_{n}(t)$ joining the basepoint $X$ to the corresponding maximal cusp $C_{n, k_{n}}$ stays in a uniform neighborhood of $r_{n}$ and has length going to $\infty$. If $\hat{r}$ is a finite ray defined on $[0, T]$ then the fact that the completion is $\operatorname{CAT}(0)$ implies that $X_{n}(T) \rightarrow \widehat{r}(T)$. Now the same argument as in the proof of surjectivity gives a contradiction. This finishes the proof.

\section{Non-relative hyperbolicity}

In this section we address the question of the strong relative hyperbolicity of $P(S)$ and the Weil-Petersson metric on $\operatorname{Teich}(S)$ when $\zeta(S)>3$. We will borrow extensively from the ideas and terminology of [2], who show that for surfaces $S$ with $\zeta(S) \geq 6$ that $P(S)$ is thick, a condition which will, in this context, guarantee that $P(S)$ is not strongly relatively hyperbolic with respect to any collection of co-infinite subsets (a 
subset of a metric space is co-infinite if there are points in the space at an arbitrarily large distance from the set).

For the purposes of exposition, we say $S$ is of mid-range complexity if $\zeta(S) \in\{4,5\}$, in other words, $S=S_{g, n}$ and we have

$$
(g, n) \in\{(0,7),(0,8),(1,4),(1,5),(2,1),(2,2)\} .
$$

Definition 16 A curve $\gamma$ is said to be domain separating if it separates $S$ into two components $Y$ and $Y^{c}$, neither of which is a 3 -holed sphere.

Accordingly, let $\mathcal{C}_{\text {sep }}(S) \subset \mathcal{C}(S)$ be the set of domain separating curves.

If $\gamma \in \mathcal{C}_{\text {sep }}(S)$ and $Y_{1}$ and $Y_{2}$ are disjoint subsurfaces of $S \backslash \gamma$ with $\zeta\left(Y_{i}\right) \geq 1, i=1,2$, any two hierarchy paths $\rho_{1}$ and $\rho_{2}$ in $P\left(Y_{1}\right)$ and $P\left(Y_{2}\right)$ determine a quasi-flat

$$
\rho_{1} \times \rho_{2}: \mathbb{Z} \times \mathbb{Z} \rightarrow P(S)
$$

namely, quasi-isometrically embedding $\mathbb{Z} \times \mathbb{Z}$ in $P(S)$ with constants not depending on $\gamma, \rho_{1}$ or $\rho_{2}$ (this was observed in [7] - it follows from the distance formula (2-1)). In [2] a general definition is given for a collection of metric spaces to be uniformly thick of order at most $n+1$.

Definition 17 [2, Definition 7.1] A metric space is thick of order zero if it is unconstricted.

A metric space is thick of order at most $n+1$ with respect to a collection $\mathcal{L}$ of subsets of $X$ if:

- With their restricted metric from $X$, the subsets in $\mathcal{L}$ are uniformly thick of order $n$.

- For some fixed $r>0$,

$$
X=\bigcup_{L \in \mathcal{L}} \mathcal{N}_{r}(L)
$$

- Any two elements $L$ and $L^{\prime}$ in $\mathcal{L}$ can be thickly connected; there exists a sequence $L=L_{1}, L_{2}, \ldots, L_{m}=L^{\prime}$ with $L_{i} \in \mathcal{L}$ and with

$$
\operatorname{diam}\left(\mathcal{N}_{r}\left(L_{i}\right) \cap \mathcal{N}_{r}\left(L_{i+1}\right)\right)=\infty
$$

for all $1 \leq i \leq m-1$.

A collection $\left\{X_{i}\right\}$ is called uniformly thick of order at most $n+1$ if a uniform $r$ can be taken in the above definition. 
The condition that a metric space be unconstricted makes use of the asymptotic cone of a metric space, which will not be necessary for our considerations. We will work instead with uniform quasi-flats discussed above, which are themselves uniformly unconstricted (see Behrstock, Druţu and Mosher [2]).

Theorem 18 If $\zeta(S) \in\{4,5\}$ and $S \neq S_{2,1}$ then $P(S)$ is thick of order 1 . If $S=S_{2,1}$ then $P(S)$ is thick of order at most 2 .

In [2] the first statement of the theorem is established for $P(S)$ when $\zeta(S) \geq 6$, as well as for the mapping class group when $\zeta(S) \geq 2$.

As in what follows, their proof is based on finding thickly connected chains of quasi-flats. In our cases, the existence of such chains relies on a detailed study of the connectivity of the subcomplex of domain separating curves $\mathcal{C}_{\text {sep }}(S) \subset \mathcal{C}(S)$.

Lemma 6 Let $S=S_{g, n}$, where $(g, n) \in\{(1,4),(1,5),(0,7),(0,8)\}$. Then $\mathcal{C}_{\text {sep }}(S)$ is connected.

Proof Let $\alpha$ and $\beta$ lie in $\mathcal{C}_{\text {sep }}(S)$. It suffices either to find $\gamma \in \mathcal{C}_{\text {sep }}(S)$ disjoint from $\alpha$ such that $i(\gamma, \beta)<i(\alpha, \beta)$, or to replace $\alpha$ with $\alpha^{\prime} \in \mathcal{C}_{\text {sep }}(S)$ disjoint from $\alpha$ so that $i\left(\alpha^{\prime}, \beta\right)=i(\alpha, \beta)$ and then find such a $\gamma$. For then in at most two steps we have reduced intersection numbers, and inductively we can find the desired path.

We now consider the cases of $S_{1,4}$ and $S_{0,7}$. Let $\eta$ be an arc in the complement of $\alpha$ joining $\beta$ to the puncture $p$. Then we say $\beta^{\prime}$ is obtained from $\beta$ by moving the puncture $p$ across $\beta$ along $\eta$ if $\beta^{\prime}$ is the component of the boundary of a regular neighborhood of $\beta \cup \eta$ not isotopic to $\beta$.

Start with the case $S=S_{1,4}$. Given $\alpha$ and $\beta$ in $\mathcal{C}_{\text {sep }}(S)$ we may move a puncture across one of these along an arc in the complement of the other if necessary to arrange that they enclose different numbers of punctures, either four or three. Without loss of generality assume that $S \backslash \alpha$ contains a subsurface $Y$ containing four punctures.

We claim that not all arcs of $\beta \cap Y$ with endpoints on $\alpha$ can lie in the homotopy class $\bmod \alpha$ that separates the four punctures in $Y$ into two pairs of punctures. For each pair lies in a single component of the complement of $\beta$ one of which must contain three punctures by hypothesis. Thus there must be a homotopy class of arcs in $Y$ that has exactly 3 punctures in its complement, and now a surgery produces a curve $\gamma$ disjoint from $\alpha$.

For $S=S_{0,7}$ every curve in $\mathcal{C}_{\text {sep }}(S)$ divides the surface into two components, one of which contains three punctures and one of which contains four punctures. Let $Y$ be 
the component of $S \backslash \alpha$ that contains four punctures. If some arc of $\beta \cap Y$ separates one puncture from the other three, we may perform a surgery as above. Thus assume each arc of $\beta \cap Y$ separates the punctures in $Y$ into two pairs of punctures. One pair lies in the component of the complement of $\beta$ containing four punctures.

There are two cases. In the first case, all four of the punctures in $Y$ are in the same component of the complement of $\beta$. In that case we can move any of the punctures across $\alpha$, or equivalently find a disjoint $\alpha^{\prime}$. Then the three remaining punctures in the complement of $\alpha^{\prime}$ are in the same component of the complement of $\beta$ as the one moved puncture which is now in the component of the complement of $\alpha^{\prime}$ containing four punctures. The other three punctures are in the other component of the complement of $\beta$, and thus the moved puncture is separated by an arc from those punctures. We have reduced to the case where we can now perform a surgery.

In the second case, a pair of punctures in $Y$ are in the same component of the complement of $\beta$ as a pair of punctures in the complement of $Y$. We now move one of these punctures in $Y$ across $\alpha$ forming $\alpha^{\prime}$. Now there are three punctures in the component of the complement of $\alpha^{\prime}$ that contains four punctures that are in the same component of the complement of $\beta$ and again we can perform the surgery.

The cases $S=S_{0,8}$ and $S=S_{1,5}$ follow readily from the observation that filling in a puncture gives a well defined map from

$$
\mathcal{C}_{\text {sep }}\left(S_{g, n}\right) \rightarrow \mathcal{C}\left(S_{g, n-1}\right)
$$

whose image lies in $\mathcal{N}_{1}\left(\mathcal{C}_{\text {sep }}\left(S_{g, n-1}\right)\right)$ and contains $\mathcal{C}_{\text {sep }}\left(S_{g, n-1}\right)$.

Thus, given a pair of curves in $\mathcal{C}_{\text {sep }}\left(S_{1,5}\right)$ we may find separating curves at distance 1 from these whose images lie in $\mathcal{C}_{\text {sep }}\left(S_{1,4}\right)$ after filling in the appropriate puncture, and similarly for $S_{0,8}$. These cases of the lemma then follow from the connectivity of $\mathcal{C}_{\text {sep }}\left(S_{1,4}\right)$ and $\mathcal{C}_{\text {sep }}\left(S_{0,7}\right)$.

Lemma 7 Let $S=S_{2,2}$. Given $\gamma_{1}, \gamma_{N} \in \mathcal{C}_{\text {sep }}(S)$ there is a sequence $\gamma_{1}, \gamma_{2}, \ldots, \gamma_{N}$ of curves in $\mathcal{C}_{\text {sep }}(S)$ such that for each $i \leq N-1$, either $\gamma_{i}$ and $\gamma_{i+1}$ are disjoint or $S \backslash\left(\gamma_{i} \cup \gamma_{i+1}\right)$ is a sphere with 4 punctures.

Proof By filling in the punctures we can consider the curves as lying on a closed surface. By a result of Schleimer [23] (see also Putman [21]) on a closed surface of genus 2 there is a sequence $\gamma_{1}, \ldots, \gamma_{N}$ of separating curves such that successive curves intersect minimally, which means four times. This implies that some complementary component of their union is an annulus. Since on the punctured surface we may move the punctures across separating curves so that they both lie in one of these 
complementary annuli, we can produce a sequence of separating curves satisfying the conditions of the Lemma.

Now suppose $S$ is any surface of mid-range complexity other than $S_{2,1}$. If $Y \subset S$ is a proper essential subsurface with $\zeta(Y) \geq 1$ and $\rho(n)$ is a hierarchy path in $P(Y)$, we denote by $|\rho(n)|$ the collection of curves in the pants decomposition $\rho(n)$ together with components of $\partial Y$ that are nonperipheral in $S$.

If $Y_{1}$ and $Y_{2}$ are disjoint essential subsurfaces of $S$ and $\rho_{1}$ and $\rho_{2}$ are bi-infinite hierarchy paths in $P\left(Y_{1}\right)$ and $P\left(Y_{2}\right)$ for which the union $\left|\rho_{1}(n)\right| \cup\left|\rho_{2}(m)\right|$ (forgetting possible repetitions of curves) is a pants decomposition of $S$, then we denote by

$$
\mathcal{Q}_{\rho_{1}, \rho_{2}}: \mathbb{Z} \times \mathbb{Z} \rightarrow P(S)
$$

the natural quasi-flat determined by

$$
\mathcal{Q}_{\rho_{1}, \rho_{2}}(m, n)=\left|\rho_{1}(n)\right| \cup\left|\rho_{2}(m)\right| .
$$

Given $\gamma \in \mathcal{C}_{\text {sep }}(S)$, we let $\mathcal{L}_{\gamma}$ denote all such quasi-flats $\mathcal{Q}_{\rho_{1}, \rho_{2}}$ with image in $X_{\gamma}$ (so that $\gamma$ lies in each pants decomposition in the image of $\mathcal{Q}_{\rho_{1}, \rho_{2}}$ ). Finally, let $\mathcal{L}$ denote the union of all quasi-flats in all $\mathcal{L}_{\gamma}$, in other words

$$
\mathcal{L}=\left\{\mathcal{Q} \in \mathcal{L}_{\gamma}: \gamma \in \mathcal{C}_{\text {sep }}(S)\right\}
$$

As we have remarked before, there are constants $K>1$ and $C>0$ such that each $\mathcal{Q}$ in $\mathcal{L}$ is $(K, C)$-quasi-isometrically embedded into $P(S)$, so the collection of quasi-flats $\mathcal{L}$ is uniformly thick of order 0 , in the sense of [2].

Lemma 8 If $S$ has mid-range complexity and $S \neq S_{2,1}$ then every $P \in P(S)$ is within distance 1 of an element in $\mathcal{L}$.

Proof Given a $\gamma \in \mathcal{C}_{\text {sep }}(S)$ and a pants decomposition $P$ containing $\gamma$, we can find an element of $\mathcal{L}_{\gamma}$ containing $P$ : this amounts to observing that there are bi-infinite hierarchy paths through any point in $P(Y)$ for $Y$ a component of $S \backslash \gamma$. Thus, it suffices to show that each pants decomposition $P \in P(S)$ lies within distance 1 of some $P^{\prime}$ containing a separating curve.

In the case of the sphere it is obvious that every pants decomposition contains a curve in $\mathcal{C}_{\text {sep }}(S)$ so we consider the case of $S_{1,4}$. We can assume that there are curves $\beta_{1}$ and $\beta_{2}$ in $P$ surrounding a pair of punctures each; otherwise we would be done. The complement of $\beta_{1} \cup \beta_{2}$ is a torus $Z$ with 2 punctures. If there is a curve in $Z$ that bounds a punctured torus we again are done; so assume otherwise. This means that there are curves $\beta_{3}$ and $\beta_{4}$ in $P$ which each cut $Z$ into a 4 holed sphere. An 
elementary move now changes one of these into a pants decomposition containing a curve that bounds a punctured torus.

In the case of $S_{1,5}$ we can again assume the existence of $\beta_{1}$ and $\beta_{2}$ as in the previous case. The complement now is a torus $Z$ with 3 holes. If there is a curve that surrounds both $\beta_{1}$ and $\beta_{2}$ or surrounds one of these curves and the remaining puncture $x$, we are finished. If there is a curve which cuts off a punctured torus, we are again finished. Thus, we assume the remaining possibility holds: there is a curve $\beta_{3} \in P$ which cuts $Z$ into a 5 holed sphere $W$. We can now assume there is a curve $\beta_{4} \in P$ which cuts $W$ into a 4 holed sphere $V$ and a 3 -holed sphere, and so that the union $\beta_{3} \cup \beta_{4}$ separates $Z$. Without loss of generality we can assume $\beta_{1}$ is a boundary curve of $V$. Inside $V$ there is a last curve $\beta_{5} \in P$ which separates $x$ from $\beta_{1}$; for otherwise we would be done. Now an elementary move inside $V$ replaces $\beta_{5}$ with one that contains $x$ and $\beta_{1}$, and hence lies in $\mathcal{C}_{\text {sep }}(S)$.

The case of $S_{2,2}$ is easier since in the closed genus 2 every pants decomposition is distance at most 1 from one containing a separating curve.

The proof that $P(S)$, for $S \neq S_{2,1}$, is thick of order at most 1 is concluded by:

Proposition 2 Any two quasi-flats $\mathcal{Q}$ and $\mathcal{Q}^{\prime}$ in $\mathcal{L}$ can be thickly connected: there exists a sequence

$$
\mathcal{Q}=\mathcal{Q}_{1}, \ldots, \mathcal{Q}_{N}=\mathcal{Q}^{\prime}
$$

with $\mathcal{Q}_{i} \in \mathcal{L}$, and for some fixed $r>0$,

$$
\operatorname{diam}\left(\mathcal{N}_{r}\left(\mathcal{Q}_{i}\right) \cap \mathcal{N}_{r}\left(\mathcal{Q}_{i+1}\right)\right)=\infty
$$

for all $1 \leq i \leq N-1$.

Proof Consider $S_{g, n}$ where $(g, n) \in\{(0,7),(0,8),(1,4),(1,5)\}$. The proof of the Proposition in these cases follows from following two observations:

(1) Given $\gamma \in \mathcal{C}_{\text {sep }}(S)$, and quasi-flats $\mathcal{Q}=\mathcal{Q}_{\rho_{1}, \rho_{2}}$ and $\mathcal{Q}^{\prime}=\mathcal{Q}_{\rho_{1}, \rho_{2}^{\prime}}$ in $\mathcal{L}_{\gamma}$, there is a quasi-flat $\mathcal{Q}^{\prime \prime}=\mathcal{Q}_{\rho_{1}, \rho_{2}^{\prime \prime}}$ in $\mathcal{L}_{\gamma}$ so that

$$
\operatorname{diam}\left(\mathcal{Q} \cap \mathcal{Q}^{\prime \prime}\right)=\infty \text { and } \operatorname{diam}\left(\mathcal{Q}^{\prime \prime} \cap \mathcal{Q}^{\prime}\right)=\infty
$$

(2) Given any disjoint pair $\gamma$ and $\gamma^{\prime}$ in $\mathcal{C}_{\text {sep }}(S)$, there is a quasi-flat $\mathcal{Q}$ in $P(S)$ so that $\mathcal{Q} \in \mathcal{L}_{\gamma} \cap \mathcal{L}_{\gamma^{\prime}}$ 
To see the first statement, we need only observe that if $Y$ is the component of $S \backslash \gamma$ containing $\left|\rho_{2}(0)\right|$ and $\left|\rho_{2}^{\prime}(0)\right|$ there is a bi-infinite hierarchy path $\rho_{2}^{\prime \prime}$ in $P(Y)$ so that $\rho_{2}^{\prime \prime}(0)=\rho_{2}(0)$ and $\rho_{2}^{\prime \prime}(j)=\rho_{2}^{\prime}(0)$ for some $j$. Then we have

$$
\mathcal{Q}(\mathbb{Z} \times\{0\})=\mathcal{Q}^{\prime \prime}(\mathbb{Z} \times\{0\}) \text { and } \mathcal{Q}^{\prime \prime}(\mathbb{Z} \times\{j\})=\mathcal{Q}^{\prime}(\mathbb{Z} \times\{0\}),
$$

so each intersection $\mathcal{Q} \cap \mathcal{Q}^{\prime \prime}$ and $\mathcal{Q}^{\prime \prime} \cap \mathcal{Q}^{\prime}$ has infinite diameter.

To see the second assertion, observe that for disjoint curves $\gamma$ and $\gamma^{\prime}$ in $\mathcal{C}_{\text {sep }}(S)$, that determine precisely two components $Z_{1}$ and $Z_{2}$ in $S \backslash \gamma \cup \gamma^{\prime}$ with $\zeta\left(Z_{i}\right) \geq 1$ for $i=1,2$, we may take $\rho_{i}$ to be a bi-infinite hierarchy path in $P\left(Z_{i}\right)$, and the quasi-flat $\mathcal{Q}_{\rho_{1}, \rho_{2}}$ lies in $\mathcal{L}_{\gamma} \cap \mathcal{L}_{\gamma^{\prime}}$, satisfying the claim. If $S \backslash \gamma \cup \gamma^{\prime}$ has three components each with complexity 1 , there is a third curve $\gamma^{\prime \prime} \in \mathcal{C}_{\text {sep }}(S)$ disjoint from $\gamma$ and $\gamma^{\prime}$ and separating them, so that taking $Z_{i}$ to be the two components of $S \backslash \gamma \cup \gamma^{\prime}$ not containing $\gamma^{\prime \prime}$ and $\rho_{1}$ and $\rho_{2}$ as before, we have a hierarchy path

$$
\rho_{2}^{\prime}(n)=\left|\rho_{2}(n)\right| \cup \gamma^{\prime \prime}
$$

for which the quasi-flat $\mathcal{Q}_{\rho_{1}, \rho_{2}^{\prime}}$ satisfies the claim.

When $S=S_{2,2}$ and $\mathcal{C}_{\text {sep }}(S)$ is not necessarily connected, we replace condition (2) with:

$\left(2^{\prime}\right)$ If $S=S_{2,2}$ and $\gamma$ and $\gamma^{\prime}$ in $\mathcal{C}_{\text {sep }}(S)$ intersect in such a way that $S \backslash \gamma \cup \gamma^{\prime}$ contains a 4-holed sphere $Z$, then there is an $r>0$ and quasi-flats $\mathcal{Q} \in \mathcal{L}_{\gamma}$ and $\mathcal{Q}^{\prime} \in \mathcal{L}_{\gamma^{\prime}}$ for which

$$
\operatorname{diam}\left(\mathcal{N}_{r}(\mathcal{Q}) \cap \mathcal{N}_{r}\left(\mathcal{Q}^{\prime}\right)\right)=\infty .
$$

To see this, let $Y$ be the component of $S \backslash \gamma$ not containing $Z$ and let $Y^{\prime}$ be the component of $S \backslash \gamma^{\prime}$ not containing $Z$. Let $\rho_{1}$ be any bi-infinite hierarchy path in $P(Z)$. Taking $\rho_{2}$ in $P(Y)$ and $\rho_{2}^{\prime}$ in $P\left(Y^{\prime}\right)$ to be bi-infinite hierarchy paths, we obtain quasi-flats

$$
\mathcal{Q}=\mathcal{Q}_{\rho_{1}, \rho_{2}} \text { and } \mathcal{Q}^{\prime}=\mathcal{Q}_{\rho_{1}, \rho_{2}^{\prime}} .
$$

Then letting $r$ be the distance between $\mathcal{Q}(0,0)$ and $\mathcal{Q}^{\prime}(0,0)$, we see that

$$
\operatorname{diam}\left(\mathcal{N}_{r}(\mathcal{Q}(\mathbb{Z} \times\{0\})) \cap \mathcal{N}_{r}\left(\mathcal{Q}^{\prime}(\mathbb{Z} \times\{0\})\right)\right)=\infty .
$$

To prove the claim, we need only observe that the configuration of $\gamma$ and $\gamma^{\prime}$ on $S$ is unique up to homeomorphisms of $S$ to see that $r$ can be taken independently of the pair of curves $\gamma$ and $\gamma^{\prime}$.

Applying Lemma 7, we may find a sequence of curves $\left\{\gamma_{i}\right\}$ in $\mathcal{C}_{\text {sep }}(S)$ satisfying the conclusions of the lemma. Conditions (1), (2) and $\left(2^{\prime}\right)$ guarantee that we can 
thickly connect quasi-flats in each $\mathcal{L}_{\gamma_{i}}$ to join quasi-flats in $\mathcal{L}_{\gamma_{i}}$ that thickly connect to quasi-flats in $\mathcal{L}_{\gamma_{i-1}}$ and $\mathcal{L}_{\gamma_{i+1}}$. The Proposition follows.

In [1] it was shown that $P(S)$ is not unconstricted, which, in particular, guarantees that $P(S)$ cannot be thick of order zero. It follows that in the cases above that $P(S)$ is thick of order exactly 1 (we thank Jason Behrstock for alerting us to this point).

We now consider the remaining case.

Proposition 3 Let $S^{\prime}=S_{2,1}$. The pants graph $P\left(S^{\prime}\right)$ is thick of order at most 2 .

Proof Let $x$ denote the puncture on $S^{\prime}$ and let $S$ be the closed surface of genus 2 obtained by adding $x$ to $S^{\prime}$. It is well known that there exists an injective homomorphism

$$
\pi_{1}(S, x) \rightarrow \operatorname{Mod}\left(S^{\prime}\right)
$$

obtained by "pushing $x$ around a loop." By a theorem of Kra [18], if $\gamma \in \pi_{1}(S, x)$ has positive geometric intersection with every essential nonperipheral simple closed curve ( $\gamma$ is filling), then its image under this homomorphism is a pseudo-Anosov diffeomorphism. Let $G$ be the image of $\pi_{1}(S, x)$.

Filling in the puncture also induces a map

$$
\Pi: \mathcal{C}\left(S^{\prime}\right) \rightarrow \mathcal{C}(S)
$$

The action of $G$ preserves each fiber $\Pi^{-1}(\alpha) ; \alpha \in \mathcal{C}(S)$. It is known [23, Proposition 4.3] that the fibers $\Pi^{-1}(\alpha)$ are connected. Given $\gamma \in \mathcal{C}(S)$, let $\widehat{X}_{\gamma}$ be the collection of pants decompositions in $P\left(S^{\prime}\right)$ so that each $P \in \widehat{X}_{\gamma}$ contains some $\gamma^{\prime} \in \Pi^{-1}(\gamma)$.

Lemma 9 Let $\gamma \in \mathcal{C}_{\text {sep }}(S)$. Then $\widehat{X}_{\gamma}$ is thick of order at most 1 and the collection $\mathcal{L}$ of all $\widehat{X}_{\gamma}$ for $\gamma \in \mathcal{C}_{\text {sep }}(S)$ is uniformly thick.

Proof Each $\gamma^{\prime} \in \Pi^{-1}(\gamma)$ divides the surface $S^{\prime}$ into subsurfaces $Y_{1}$ and $Y_{2}$ with $\zeta\left(Y_{i}\right) \geq 1$. Taking hierarchy paths $\rho_{i}$ in $P\left(Y_{i}\right)$ and quasi-flats $\mathcal{Q}_{\rho_{1}, \rho_{2}}$ as before, connectedness of the fiber allows us to argue using conditions (1) and (2) from the previous Proposition that for any two curves $\gamma^{\prime}$ and $\gamma^{\prime \prime}$ in the fiber $\Pi^{-1}(\gamma)$, quasi-flats in $\mathcal{L}_{\gamma^{\prime}}$ and $\mathcal{L}_{\gamma^{\prime \prime}}$ can be thickly connected within $\widehat{X}_{\gamma}$.

Since each $P \in \widehat{X}_{\gamma}$ lies in some quasi-flat of this form, we have $\widehat{X}_{\gamma}$ is thick of order 1 . Since the constants do not depend on $\gamma$, the union $\mathcal{L}$ is a collection of uniformly thick subsets of $P\left(S^{\prime}\right)$. 
We now conclude the proof that $P\left(S^{\prime}\right)$ is thick of order at most 2 .

Exactly as in the case of $S_{2,2}$, any point in $P\left(S^{\prime}\right)$ is within distance at 1 of a point in $\mathcal{L}$. Now we show that any two elements of $\mathcal{L}$ can be thickly connected. Given $\gamma_{1}$ and $\gamma_{N}$ in $\mathcal{C}_{\text {sep }}\left(S^{\prime}\right)$, we join them by a sequence $\gamma_{1}, \ldots, \gamma_{N}$ in $\mathcal{C}_{\text {sep }}\left(S^{\prime}\right)$ where successive curves are either disjoint or intersect minimally. There is a uniform constant $C^{\prime}$ and pants decompositions, $P_{i}$ that contain $\gamma_{i}$, such that

$$
d\left(P_{i}, P_{i+1}\right) \leq C^{\prime} .
$$

Now let $\phi$ be a pseudo-Anosov element in $G$. The orbit

$$
\left\{\phi^{n}\left(P_{i}\right)\right\}_{n=-\infty}^{\infty}
$$

is an infinite diameter subset of $\widehat{X}_{\gamma_{i}}$. As $\phi$ acts isometrically on $P\left(S^{\prime}\right)$, we have

$$
\begin{aligned}
d\left(\phi^{n}\left(P_{i}\right), \phi^{n}\left(P_{i+1}\right)\right) & =d\left(P_{i}, P_{i+1}\right) \\
& \leq C^{\prime} .
\end{aligned}
$$

This guarantees that $\widehat{X}_{\gamma_{1}}$ and $\widehat{X}_{\gamma_{N}}$ can be thickly connected.

Proof of Theorem 6 By Theorem 18 (for $\zeta(S)=4$ and 5) and [2, Corollary 7.9] the pants graph cannot be asymptotically tree-graded for each $S$ with $\zeta(S) \geq 4$.

The theorem then follows immediately from the equivalence of strong relative hyperbolicity with the condition that a metric space is asymptotically tree-graded [11, Theorem 4.1].

Remark It is interesting to note that the proof of Theorem 6 does not show that $P\left(S_{2,1}\right)$ is thick of order exactly 2 . It would be interesting to know whether $P\left(S_{2,1}\right)$ presents such a special case.

\section{References}

[1] J A Behrstock, Asymptotic geometry of the mapping class group and Teichmüller space, Geom. Topol. 10 (2006) 1523-1578 MR2255505

[2] J A Behrstock, C Druţu, L Mosher, Thick metric spaces, relative hyperbolicity, and quasi-isometric rigidity, submitted

[3] J A Behrstock, Y Minsky, Dimension and rank for mapping class groups, Ann. of Math. (2) 167 (2008) 1055-1077

[4] MR Bridson, A Haefliger, Metric spaces of non-positive curvature, Grund. der Math. Wissenschaften [Fund. Princ. of Math. Sciences] 319, Springer, Berlin (1999) MR1744486 
[5] J F Brock, The Weil-Petersson metric and volumes of 3-dimensional hyperbolic convex cores, J. Amer. Math. Soc. 16 (2003) 495-535 MR1969203

[6] J F Brock, The Weil-Petersson visual sphere, Geom. Dedicata 115 (2005) 1-18 MR2180039

[7] J F Brock, B Farb, Curvature and rank of Teichmüller space, Amer. J. Math. 128 (2006) 1-22 MR2197066

[8] J F Brock, H A Masur, Y N Minsky, Asymptotics of Weil-Petersson geodesics I: ending laminations, recurrence, and flows arXiv:0802.1370

[9] G Daskalopoulos, R Wentworth, Classification of Weil-Petersson isometries, Amer. J. Math. 125 (2003) 941-975 MR1993745

[10] C Druţu, Relatively hyperbolic groups: geometry and quasi-isometric invariance arXiv:mathGT/0605211

[11] C Druţu, M Sapir, Tree-graded spaces and asymptotic cones of groups, Topology 44 (2005) 959-1058 MR2153979 With an appendix by Denis Osin and Sapir

[12] A Eskin, B Farb, Quasi-flats and rigidity in higher rank symmetric spaces, J. Amer. Math. Soc. 10 (1997) 653-692 MR1434399

[13] B Farb, Relatively hyperbolic groups, Geom. Funct. Anal. 8 (1998) 810-840 MR1650094

[14] A Hatcher, W Thurston, A presentation for the mapping class group of a closed orientable surface, Topology 19 (1980) 221-237 MR579573

[15] Y Imayoshi, M Taniguchi, An introduction to Teichmüller spaces, Springer, Tokyo (1992) MR1215481 Translated and revised from the Japanese by the authors

[16] E Klarreich, The boundary at infinity of the curve complex and the relative Teichmüller space, preprint (1999)

[17] B Kleiner, B Leeb, Rigidity of quasi-isometries for symmetric spaces and Euclidean buildings, Inst. Hautes Études Sci. Publ. Math. (1997) 115-197 MR1608566

[18] I Kra, On the Nielsen-Thurston-Bers type of some self-maps of Riemann surfaces, Acta Math. 146 (1981) 231-270 MR611385

[19] H A Masur, Y N Minsky, Geometry of the complex of curves. I. Hyperbolicity, Invent. Math. 138 (1999) 103-149 MR1714338

[20] H A Masur, Y N Minsky, Geometry of the complex of curves. II. Hierarchical structure, Geom. Funct. Anal. 10 (2000) 902-974 MR1791145

[21] A Putman, A note on the connectivity of certain complexes associated to surfaces, preprint

[22] K Rafi, A combinatorial model for the Teichmüller metric, Geom. Funct. Anal. 17 (2007) 936-959 MR2346280 
[23] S Schleimer, Notes on the complex of curves, unpublished

[24] S A Wolpert, Geodesic length functions and the Nielsen problem, J. Differential Geom. 25 (1987) 275-296 MR880186

[25] S A Wolpert, Geometry of the Weil-Petersson completion of Teichmüller space, from: "Surveys in differential geometry, Vol. VIII (Boston, MA, 2002)", Surv. Differ. Geom. VIII, Int. Press, Somerville, MA (2003) 357-393 MR2039996

Department of Mathematics, Brown University

Box 1917, Providence, RI 02912

Department of Mathematics, UIC

Chicago, IL 60607

brock@math.brown.edu, masur@math.uic.edu

http://www.math.brown.edu/ brock, http://www.math.uic.edu/ masur

Proposed: Walter Neumann

Seconded: Benson Farb, Dave Gabai
Received: 12 July 2007

Revised: 14 September 2008 